Predicting Partial Atomic Charges in Siliceous Zeolites

Peer-reviewed author version

Wolffis, Jarod J.; VANPOUCKE, Danny E.P.; Sharma, Amit; Lawler, Keith V. \&

Forster, Paul M. (2019) Predicting Partial Atomic Charges in Siliceous Zeolites. In:

MICROPOROUS AND MESOPOROUS MATERIALS, 277, p. 184-196.

DOI: $10.1016 /$ j.micromeso.2018.10.028

Handle: http://hdl.handle.net/1942/27472 


\title{
Predicting Partial Atomic Charges in Siliceous Zeolites
}

\author{
Jarod J. Wolffis ${ }^{1}$, Danny E. P. Vanpoucke ${ }^{2}$, Amit Sharma ${ }^{1}$, Keith V. Lawler ${ }^{1, *}$, Paul M. \\ Forster $^{1, *}$ \\ ${ }^{1}$ University of Nevada Las Vegas, Department of Chemistry and Biochemistry, Las Vegas, NV 89154-4003, \\ USA \\ ${ }^{2}$ UHasselt, Institute for Materials Research (IMO-IMOMEC), Agoralaan, 3590 Diepenbeek, Belgium \\ *To whom correspondence should be addressed, email: keith.lawler@unlv.edu, Paul.Forster@unlv.edu
}

\begin{abstract}
$\underline{\text { Abstract }}$
Partial atomic charge, which determines the magnitude of the Coulombic non-bonding interaction, represents a critical parameter in molecular mechanics simulations. Partial charges may also be used as a measure of physical properties of the system, ie. covalency, acidic/catalytic sites, etc. A range of methods, both empirical and ab initio, exist for calculating partial charges in a given solid, and several of them are compared here for siliceous (pure silica) zeolites. The relationships between structure and the predicted partial charge are examined. The predicted partial charges from different methods are also compared with related experimental observations, showing that a few of the methods offer some guidance towards identifying the T-sites most likely to undergo substitution or for proton localization in acidic framework forms. Finally, we show that assigning unique calculated charges to crystallographically unique framework atoms makes an appreciable difference in simulating predicting $\mathrm{N}_{2}$ and $\mathrm{O}_{2}$ adsorption with common dispersion-repulsion parameterizations.
\end{abstract}

\section{$\underline{\text { Keywords }}$}

Zeolite, Partial Charge, Molecular Mechanics, DFT, T-atom substitution, Acid Catalysis 


\section{$\underline{1 \text { Introduction }}$}

Zeolites represent the most commercially important class of crystalline nanoporous materials, finding application in catalysis, adsorption, molecular sieving, ion exchange, and electronics.[1-13] The International Zeolite Association (IZA) currently recognizes over 230 unique zeolite topologies.[14] The majority of these may be synthesized over a range of $\mathrm{Si} / \mathrm{Al}$ ratios, may contain additional T-site substitution (ie., Ge, Sn, Ti, P), and ionexchanged to contain a range of cations. All these substitutions modify effective pore size and surface chemistry which, in turn, affects a specific zeolites performance for specific applications. Considering the diversity of potential zeolites and their potential for applications, appreciable development has occurred towards developing simulation approaches to aid in understanding and predicting zeolite behavior. The majority of these studies have focused on force field-based molecular mechanics approaches.

Reliable simulations must accurately describe a zeolite's structure and correctly model the interaction between the framework atoms with guest species. The chief commonality between force fields developed for these purposes is the inclusion of a Coulomb term utilizing partial charges located on the framework atom centers. Following the work of Catlow et al.,[15,16] many popular structural force fields use formal ionic charges at each atom center ( +4 for $\mathrm{Si}$ and -2 for $\mathrm{O}$ ) with a core-shell term on the oxygen atoms.[17] As on-site ion polarization and charge transfer between ions are nearly indistinguishable, the core-shell term can be thought of as describing either the polarizability of the oxygen or the covalency between the framework atoms.[18] Other force fields geared towards modeling structure instead use no core-shell term and smaller partial atomic charges, accounting for covalency with dispersion-repulsion terms or 
conventional harmonic bonding potentials.[19-24] Force fields developed expressly for separation and sieving applications (such as adsorption and diffusion) do not typically include core-shell terms. If partial charges have been parameterized for the framework, they are often much smaller than those used in a structural force field.[25,26] If no partial charges have been specified, then they must be determined through various approaches, as detailed in the following sections.

For adsorption and diffusion problems, the simplest and most widely used method is to treat the framework atoms as rigid (ie. fixed positions) as this greatly reduces the computational complexity of the simulation.[27] However, it is known that zeolites can exhibit appreciable flexibility, in part due to the small energetic penalty associated with changing the T-O-T angle.[4,24] Recent experiments and simulations have shown that flexibility must be addressed in at least some instances, such as the important commercial zeolite MFI (ZSM-5), in order to obtain reasonable accuracy.[28-30] Many of the better simulations currently being published address this flexibility,[31-34] however the simulation force field now needs to describe both the framework structure and interaction with guest species well.

Another key industrial use of zeolites is as solid acid catalysts where confinement within nanopores allows for size and shape selectivity. Coupled with the possibility of high acidity, tunability in the number of (and, consequently, spacing between) acid sites, zeolites make nearly ideal acid catalysts. The catalytic site in aluminosilicates may arise from the exchange of higher valence charge-balancing cations (ie. $\mathrm{Na}^{+}$exchanged for $\mathrm{La}^{3+}$ ), creating strong local fields near the framework Al. The distance between a high valence cation site and the framework $\mathrm{Al}$ sites favors water disassociation by stabilizing hydroxyl species 
associated with the cation and $\mathrm{H}^{+}$associated with an $\mathrm{Al}^{3+}$ site, and a trivalent cation (such as $\mathrm{La}^{+3}$ ) can create two catalytic Brønsted acid sites per cation in an aluminosilicate.[35] Since strong local fields create the Brønsted acid site, it follows that the high valent cations are likely to be located at the least electropositive Al. In other words, the Al sites with the highest partial charges will likely function as the primary acid sites. Alternatively, the substitution of heavier metals, like $\mathrm{Sn}$ or $\mathrm{Ti}$ for $\mathrm{Si}$, has been shown to greatly increase the catalytic capabilities of a zeolite.[36] These heavier metals are strong Lewis acid sites and can catalyze reactions such as petroleum refining, isomerization, methanol conversion, esterification, etc.[37] Although pure $\mathrm{SiO}_{2}$ zeolites themselves are not important catalysts, many commercial catalysts have sufficiently high $\mathrm{Si} / \mathrm{Al}$ ratios that they are nearly siliceous in overall composition. Even for zeolites with smaller $\mathrm{Si} / \mathrm{Al}$ ratios, insights generated from the pure $\mathrm{SiO}_{2}$ framework often provide useful insights towards predicating catalytic sites and behavior.

Recently Hamad et al. gave an overview of many common methods and demonstrated their performance in predicting partial atomic charges for some representative metal-organic frameworks (MOFs).[38] There are very few, if any, force fields for MOFs that include a description of the partial atomic charges, so the prediction of partial atomic charges is very important for MOF simulations. They tested methods that require ab initio quantum mechanical simulations as well as those that only use a crystal structure. Their results showed a surprisingly high degree of variability in the predicted partial atomic changes depending on methodology. Not surprisingly, these differences impact separation, adsorption, and diffusion simulations. In addition, they demonstrated that the choice of 
partial atomic charge had a large impact on the predicted structural properties when framework flexibility was introduced into the simulation.

Zeolite force fields have existed for an appreciable amount of time and are far more mature than force fields for MOFs. However, existing zeolite force fields fall into two categories: 1) force fields fit to accurately account for structure in silicates (such as response to pressure) and 2) force fields designed to accurately predict adsorption in static frameworks. The treatment of partial atomic charges in these approaches tends to be quite different. With increasing computational power available, it is becoming routinely possible to carry out simulations of adsorption with framework flexibility included. A new generation of potentials are needed with physically accurate partial charges in order to simultaneously model structure and adsorption. Here, we present a study similar in spirit to that of Hamad et al. focused on understanding the predicted partial atomic charges of siliceous zeolite frameworks. Unlike MOFs, it is more difficult to break down zeolites into simple molecular clusters as the same composite building units (CBUs) appear in many zeolite frameworks and the partial charges for one CBU may vary greatly depending on which other CBUs are connected to it. There are several different methodologies that have been created to determine the partial atomic charges on atoms in a structure from structural and quantum chemical information. These methods can be applied to single molecules or periodic systems, and as our goal is to analyze their performance in zeolites the focus will be only on methods that can be applied to extended periodic systems. As many of these methods have been thoroughly discussed in the literature, we only offer a brief summary of the methods we use in Table 1 (a more comprehensive review is available in the Supplemental Information). In this work, we will compare the predicted partial atomic 
charges from each method, consider the effect of the local geometry on the charges, and we will assess if these charges can be used to identify known acidic or catalytic T-sites in select zeolites. Lastly, we will analyze the effect of compression on the predicted partial charges, and the effect the partial atomic charges from the different methodologies has on predicted gas adsorption behavior.

Table 1. A summary of the partial atomic charge determination methods used in this work.

\begin{tabular}{|c|c|c|c|}
\hline Method & Type & Description & Ref. \\
\hline EEM & $\begin{array}{l}\text { semi- } \\
\text { empirical }\end{array}$ & $\begin{array}{l}\text { Charges determined through equalization of } \\
\text { electronegativity }\end{array}$ & [39] \\
\hline QEq & $\begin{array}{l}\text { semi- } \\
\text { empirical }\end{array}$ & $\begin{array}{l}\text { Like EEM, but uses screened Coulomb repulsions } \\
\text { in lieu of atomic hardness }\end{array}$ & [40] \\
\hline EQEq & $\begin{array}{l}\text { semi- } \\
\text { empirical }\end{array}$ & $\begin{array}{l}\text { QEq which allows the Taylor series to be } \\
\text { expanded around non-zero charges }\end{array}$ & [41] \\
\hline QTPIE & $\begin{array}{l}\text { semi- } \\
\text { empirical }\end{array}$ & $\begin{array}{l}\text { QEq but expressed in terms of charge transfer } \\
\text { variables that penalize long-range charge transfer }\end{array}$ & [42] \\
\hline $\begin{array}{l}\text { Iterative } \\
\text { Hirshfeld (HI) }\end{array}$ & $a b$ initio & $\begin{array}{l}\text { Parses the charge density by fitting it to a } \\
\text { superposition of reference atomic states. }\end{array}$ & [43-45] \\
\hline DDEC & ab initio & $\begin{array}{l}\text { Atoms-in-Molecule (AIM) decomposition of } \\
\text { charge density coupled with an iterative } \\
\text { stockholder approach using the reference atomic } \\
\text { charge densities }\end{array}$ & [46-48] \\
\hline Bader & ab initio & $\begin{array}{l}\text { Atoms-in-Molecule (AIM) decomposition of } \\
\text { charge density }\end{array}$ & [49-51] \\
\hline REPEAT & ab initio & Charges fit to reproduce the electrostatic potential & [52] \\
\hline
\end{tabular}

\section{Methods}

\subsection{Simulation}

The crystal structures for the zeolites studied were taken directly from the Structure Commission of the IZA's (IZA-SC) database of structures.[14] The IZA-SC's structures, optimized with DLS-76 using only bond length and bond angle constraints, provide a fully self-consistent idealized structures appropriate as a starting point for our analysis. DLS-76 is a distance least-squared program primarily for determining zeolite structures from 
powder patterns that can enforce linear constraints on the connectivities.[53] Atomic connectivity and geometry were evaluated using the ZeoTsites package, and verified with structure visualization.[54,55] The EEM and QEq partial atomic charges were determined using the General Utility Lattice Program (GULP).[56] The EQEq partial atomic charges were determined with the stand-alone program provided as a supplement to the work of Wilmer et al.[41] The QTPIE partial atomic charges were determined with GULP.

Periodic plane-wave DFT calculations were performed with version 5.4 .1 of the Vienna ab-initio Simulations Package (VASP). The generalized gradient approximation (GGA) exchange-correlation functional of Perdew, Burke and Ernzerhof (PBE) was utilized.[57] As it has been previously demonstrated that long-range dispersion is necessary for accurately predicting zeolite frameworks, [58] Grimme's -D3 semi-empirical dispersion correction was included during structural optimizations.[59] The plane augmented wave (PAW) $[60,61]$ pseudopotentials for PBE which represent the valance configurations of $2 s^{2} 2 p^{4}$ for $\mathrm{O}$ and $3 s^{2} 3 p^{2}$ for Si were employed. Optimized cell volumes were obtained through a fit to the Vinet equation of state[62] from a series of constant volume conjugategradient optimizations of the cell shape and atomic positions that spanned at least $\pm 5 \%$ of the IZA-SC listed unit cell volumes. Constant volume optimizations were performed as two subsequent optimizations of the cell shape and lattice positions and a final single point energy evaluation were performed to minimize Pulay stress. For the optimizations only the gamma point was used to represent the first Brillouin zone. The first Brillouin zone was represented with an automatically generated $\Gamma$-centered mesh with grid points spaced evenly at $\sim 0.032 \pi \AA^{-1}$ increments along the reciprocal axes for the single point simulations used to compute the charge density and electrostatic potential. The k-space integration in 
the first Brillouin zone was done with the tetrahedron method with Blöchl corrections.[63] The plane wave basis was cut off at $520 \mathrm{eV}$. The Kohn-Sham [64] expressions were solved via the RMM-DIIS algorithm with blocked Davidson pre-convergence steps. The tolerance for energies is $10^{-5} \mathrm{eV}$. The convergence tolerance for forces was $10^{-2} \mathrm{eV} / \AA$. REPEAT partial atomic charge analysis was performed using the stand-alone package of Campañá et al.[52] Bader partial atomic charge analysis was performed using the $0.95 \mathrm{a}$ version of the Bader Charge Analysis package distributed by Henkelman et al.[65] Iterative Hirshfeld (HI) charges were determined using the HIVE program.[44,45] DDEC charges were found with the 'DDEC6' algorithm in the DDEC package distributed by Manz and Sholl.[48]

Gas adsorption was simulated with Monte Carlo in the grand canonical ensemble (GCMC) with our own in-house modified version of the MuSiC package using the PengRobinson equation of state for fugacities.[66-70] Each simulation employed 200,000 equilibration cycles and 300,000 production cycles. A cycle here consists of $\mathrm{N}$ moves, where $\mathrm{N}$ is the number of adsorbed particles (minimum 20). $2 \times 2 \times 2$ unit cells were used to represent the zeolite framework, and the framework was held as rigid during the simulations. The adsorbed particles could fluctuate with random translations, rotations, insertions, and deletions with equal weight. The adsorption energies in Table 4 were computed for a single adsorbate particle with Monte Carlo in the canonical ensemble; the particle could fluctuate with random translations, rotations, and jumps (re-insertion) with equal weight.

In the MC, the adsorbate-framework Coulomb interactions were computed using Ewald sums. On-the-fly adsorbate-adsorbate Coulomb interactions were computed using the damped, shifted potential method of Wolf cutoff at $12 \AA$ with a damping parameter, $\alpha$, 
of $0.07 \AA^{-1} ;[71]$ this methodology was chosen as we previously found it to give good agreement with on-the-fly Ewald sums.[72] The van der Waals interactions were computed with 12-6 Lennard-Jones (LJ) potentials cutoff at $20 \AA$ to minimize tail errors. The LorentzBerthelot mixing rules were used to obtain the pair-wise $\mathrm{LJ}$ parameters from the atomistic force fields. A potential energy map for adsorbate-framework interactions was made prior to the MC simulation. The framework LJ parameters were taken from the TraPPE-zeo force field.[26] The atomistic parameters for $\mathrm{N}_{2}, \mathrm{O}_{2}, \mathrm{CO}_{2}$, and $\mathrm{NH}_{3}$ are from the TraPPE-small force field,[73-75] benzene is the TraPPE united atom 9-site model,[76] and $\mathrm{H}_{2} \mathrm{O}$ is the TIP4P model.[77] The inaccessible volumes in the zeolite framework were blocked using our recently develop energy based pore mapping program with Ar as a probe $(\varepsilon=120 \mathrm{~K}$, $\sigma=3.4 \AA$ ). $[27,78]$ The simulated adsorption isotherms were excess corrected using the pore volumes produced by our energy based pore mapping program. Simulated heats of adsorption were obtained with fluctuation theory.

\subsection{Experimental}

A sample of the zeolite FAU was obtained from Joesph Hriljac that has been iteratively de-aluminated to be effectively pure $\mathrm{SiO}_{2}(\mathrm{Si} / \mathrm{Al}=\infty)$, and it has been characterized by diffraction, NMR, and compression previously.[79-81] $100 \mathrm{mg}$ of the sample was activated under dynamic vacuum at $300^{\circ} \mathrm{C}$ for 18 hours followed by a $\mathrm{N}_{2}$ surface area measurement at $77 \mathrm{~K}$. Adsorption isotherms were measured using a Micromeritics ASAP 2020 adsorption analyzer fitted with a He cryostat with a stability of $0.01 \mathrm{~K}$. All the isotherms were collected up to $\sim 750 \mathrm{mmHg}$ with incremental doses of 5 $\mathrm{cm}^{3} / \mathrm{g}$ and sufficiently long equilibration times. Desorption measurements were also taken 
at the end of each isotherm. Prior to each measurement, the sample was reactivated at $250^{\circ} \mathrm{C}$ under dynamic vacuum for an hour followed by an hour of equilibration in the cryostat at the target temperature. $\mathrm{N}_{2}$ and $\mathrm{O}_{2}$ isotherms were measured at $130 \mathrm{~K}, 140 \mathrm{~K}$ and $150 \mathrm{~K}$. Isosteric heats of adsorption were calculated by using the Clausius-Clapeyron relation.

\section{$\underline{3 \text { Results and Discussion }}$}

\subsection{Comparing Charge Methodologies}

To compare the magnitudes of the charges produced by the various methods for determining partial charges, we will focus first look at the IZA-SC structure for zeolite- $\beta$ (BEA) (data tables for the IZA-SC structures of the other known siliceous zeolites are in the SI). BEA is an important zeolite in that it has been demonstrated in the literature to be highly site-selective with regards to acid catalysis and tetrahedral atom substitution.[82] Structurally, BEA consists of three intersecting twelve membered rings (12MR) with 17 unique oxygen atoms and $9 \mathrm{~T}$ atoms. Table 2 shows the different partial charges determined for BEA by both semi-empirical and ab initio techniques broken down by its crystallographically unique atoms. Looking first at the semi-empirical techniques, we see the methods produce an increasingly ionic description of the frameworks following the order: QTPIE < QEq < EEM < EQEq. The QTPIE charges are markedly low compared to the rest of the methods. We believe they are so low because the polarization correction, although implemented for periodic boundary conditions, does not work well for extended systems. The EEM and QEq methods both produce charges that would describe a polar covalent system (ie. less than $50 \%$ ionic $\mathrm{q}_{\mathrm{Si}}=+4$ ), and that are in line with zeolite force 
fields such as that of Nicholas and TraPPE-zeo where $\mathrm{q}_{\mathrm{Si}}$ is 1.1.e and 1.5e

respectively.[20,26]

The EQEq charges are much larger than any of the other semi-empirical methods for all the zeolites studied. The EQEq charges are in line with very strong polar covalent bonds, which is the best description of the bonding in zeolite frameworks.[83] The reason the EQEq charges are much larger than the regular QEq charges is the re-centering of the Taylor expansion. Centering the charges on zero did in fact return the same charge values as QEq. Here the Taylor sum was centered around the fully ionic charges: +4 for $\mathrm{Si}$ and -2 for $\mathrm{O}$. We found that it didn't matter what value was picked for oxygen $(0,-1,-2)$; EQEq returned the same charges so long as the Taylor series for Si was centered at +4 .

Table 2. A comparison of the partial charges determined with several methodologies for the IZA-SC structure of BEA. The site 'Label' is the one used in the IZA-SC database. The average value for each atom type and the RMS deviation from that average are shown at the bottom of the table.

\begin{tabular}{|l|llllllll|}
\hline \hline Label & $\boldsymbol{E E M}$ & $\boldsymbol{Q E \boldsymbol { q }}$ & $\boldsymbol{E Q E \boldsymbol { q }}$ & $\boldsymbol{Q T P I E}$ & $\boldsymbol{H I}$ & $\boldsymbol{D D E C}$ & $\boldsymbol{B a d e r}$ & $\boldsymbol{R E P E A T}$ \\
\hline \hline O1 & -0.8066 & -0.6043 & -1.2013 & -0.0322 & -1.2786 & -1.1327 & -1.5984 & -0.5807 \\
O2 & -0.8426 & -0.6315 & -1.1570 & -0.0352 & -1.3155 & -1.0665 & -1.6150 & -0.6439 \\
O3 & -0.8046 & -0.6007 & -1.2053 & -0.0321 & -1.2827 & -1.0985 & -1.5967 & -0.5770 \\
O4 & -0.8857 & -0.6562 & -1.2921 & -0.0375 & -1.3539 & -0.9411 & -1.5885 & -0.7248 \\
O5 & -0.8857 & -0.6042 & -1.2038 & -0.0322 & -1.2778 & -1.0508 & -1.5977 & -0.5674 \\
O6 & -0.8452 & -0.6328 & -1.1638 & -0.0353 & -1.3169 & -1.0971 & -1.6153 & -0.6277 \\
O7 & -0.8073 & -0.6026 & -1.2110 & -0.0322 & -1.2853 & -1.2864 & -1.5991 & -0.5650 \\
O8 & -0.7980 & -0.5977 & -1.2103 & -0.0316 & -1.2742 & -1.6345 & -1.5942 & -0.5810 \\
O9 & -0.8646 & -0.6408 & -1.2880 & -0.0363 & -1.3355 & -0.9970 & -1.6090 & -0.6664 \\
O10 & -0.8429 & -0.6355 & -1.3070 & -0.0372 & -1.3150 & -1.0361 & -1.6020 & -0.6565 \\
O11 & -0.7974 & -0.5989 & -1.2089 & -0.0316 & -1.2757 & -1.7678 & -1.5952 & -0.5741 \\
O12 & -0.8420 & -0.6356 & -1.3043 & -0.0372 & -1.3148 & -1.0232 & -1.6008 & -0.6263 \\
O13 & -0.8413 & -0.6271 & -1.3101 & -0.0369 & -1.3171 & -1.6368 & -1.6031 & -0.6000 \\
O14 & -0.8840 & -0.6505 & -1.3959 & -0.0415 & -1.3500 & -0.9747 & -1.6128 & -0.7772 \\
O15 & -0.8527 & -0.6361 & -1.3265 & -0.0375 & -1.3229 & -1.6380 & -1.6013 & -0.6490 \\
O16 & -0.8394 & -0.6282 & -1.3046 & -0.0367 & -1.3133 & -1.3367 & -1.6031 & -0.5900 \\
O17 & -0.8493 & -0.6354 & -1.3199 & -0.0371 & -1.3190 & -1.7086 & -1.6036 & -0.6295 \\
T1 & 1.6672 & 1.2405 & 2.4059 & 0.0677 & 2.6149 & 2.1571 & 3.1964 & 1.2500 \\
T2 & 1.6680 & 1.2366 & 2.4130 & 0.0678 & 2.6153 & 2.2334 & 3.1977 & 1.2272
\end{tabular}




\begin{tabular}{|l|llllllll|} 
T3 & 1.6649 & 1.2535 & 2.4939 & 0.0670 & 2.6061 & 2.5754 & 3.2078 & 1.2656 \\
T4 & 1.6652 & 1.2528 & 2.4973 & 0.0672 & 2.6054 & 2.6140 & 3.2092 & 1.2289 \\
T5 & 1.6708 & 1.2411 & 2.6745 & 0.0808 & 2.6295 & 2.7166 & 3.1985 & 1.3045 \\
T6 & 1.6679 & 1.2439 & 2.6615 & 0.0804 & 2.6259 & 2.5198 & 3.1988 & 1.2647 \\
T7 & 1.6821 & 1.2584 & 2.5541 & 0.0670 & 2.6188 & 2.9355 & 3.2077 & 1.2233 \\
T8 & 1.6819 & 1.2655 & 2.5498 & 0.0666 & 2.6166 & 2.7506 & 3.2078 & 1.2090 \\
T9 & 1.6789 & 1.2530 & 2.5483 & 0.0677 & 2.6184 & 2.4350 & 3.2010 & 1.2636 \\
\hline \hline Avg. qO & -0.8406 & -0.6246 & -1.2594 & -0.0353 & -1.3087 & -1.2604 & -1.6021 & -0.6257 \\
RMSD & 0.0287 & 0.0185 & 0.0659 & 0.0028 & 0.0247 & 0.2869 & 0.0072 & 0.0566 \\
\hline Avg. qT & 1.6719 & 1.2495 & 2.5331 & 0.0703 & 2.6168 & 2.5486 & 3.2028 & 1.2485 \\
RMSD & 0.0067 & 0.0090 & 0.0887 & 0.0055 & 0.0075 & 0.2334 & 0.0049 & 0.0278 \\
\hline \hline
\end{tabular}

Turning to the ab initio methods, we see that the REPEAT charges are the lowest (most covalent) of the survey. The HI and DDEC methods produce very similar charges that, like EQEq, describe the strongly polar covalent bonding in the system that the coreshell potentials are built to mimic. Bader analysis produces the most ionic description of the system. Examination of how the Bader analysis approaches zeolites shows that the method produced unphysically small atomic volumes for $\mathrm{Si}$. This appears to be a buried atom problem, wherein an atom is contained within the diffuse valence shell of several other atoms making it appear that the atom has no valence charge density. Tetrahedral atoms in strongly polar covalent systems will be particularly susceptible to this problem. HI and DDEC are less prone to buried atom issues as they both use iterative comparisons to reference atomic states (neutral and ionic). The DDEC3 algorithm relies more heavily on Bader atoms-in-molecule decomposition for charge determination, and the DDEC 3 charges were much more ionic than even the Bader charges with some being too ionic: ie. $\mathrm{q}_{\mathrm{Si}}>4$, qo $<-2$. Because of this, we believe that the Bader charges are an over-estimation caused by an unphysical parsing of the charge density. Interestingly, the non-Bader ab initio methods are replicated quite well by a semi-empirical method: QEq for REPEAT and EQEq centered on the ionic charges for HI and to a lesser extent DDEC. This good 
agreement is the case for all the zeolites studied as there is a high uniformity in the magnitude of the charges predicted by a method across all the zeolites studied. Each charge determination method produced charges that vary from site-to-site on the order of $0.01 \mathrm{e}$, with the variations on the $\mathrm{O}$ atoms being greater than those of the $\mathrm{Si}$ atoms. These variations represent the different chemical environments around each unique T-site, and these site-to-site charge variations can be used to gain physical insight into the zeolite framework.

Comparison to partial atomic charge determinations from previous studies enhances our ability to understand the charges produced by the different methods. Some of the first periodic calculations on siliceous chabazite (CHA) compared the Mulliken[84] charges produced with steadily increasing basis sets with Hartree-Fock.[85] A minimal basis set yielded a charge on the Si of $2.543 \mathrm{e}$. A split valence basis saw that charge drop to $1.655 \mathrm{e}$, but adding polarization functions to the split-valence basis caused the charge to rise again to 2.230e. The largest and best basis set employed yielded a similar charge, $2.320 \mathrm{e}$, for $\mathrm{Si}$ in $\alpha$-quartz. A later study on of $\mathrm{NH}_{3}$ and $\mathrm{NH}_{4}{ }^{+}$adsorption into an aluminosilicate ( $\mathrm{Si} / \mathrm{Al}=3$ 5) CHA contrasted the use of cluster and periodic calculations for partial charges.[86] Their Milliken Si charges ranged from $1.443 \mathrm{e}$ for a periodic representation to $1.612 \mathrm{e}$ for a cluster representation. A later evaluation of Ti-containing zeolites gave 1.78, 1.67, 1.72 as the charges for $\mathrm{Si}$ in the siliceous forms of QUA, SOD, and CHA respectively.[87] The addition of Ti caused the charge on the Si to rise to $\sim 1.9 \mathrm{e}$. de Boer et al. built a force field using split-valence basis set MP2[88] calculations on silicate clusters, analyzing the Mulliken charges and also the contribution of the polarizability tensor and molecular dipole moments.[89] Their approach yielded a Mulliken charge very similar to aluminosilicate 
CHA study ( $\left.\mathrm{qSi}_{\mathrm{Si}}=1.486 \mathrm{e}\right)$, although when they factored in the polarizability and dipole moments, the charge on the Si jumped to 2.7226e. Later, Pedone et al. combined those charges with periodic B3LYP[90] simulations on dense and porous silicates to make the FFSiOH force field.[91] They kept the charges the same, but found they needed to make the oxygen atoms core-shell allowing them to fluctuate providing some fluidity to the high 2.7e charge on the Si. Recently, Fang and coworkers have worked towards developing transferable force fields for adsorption into zeolites and have used DDEC charges from accurate periodic DFT simulations, determining Si charges of 2.21e for $\mathrm{CO}_{2}$ into $\mathrm{CHA}$ [92] and 2.2124e for $\mathrm{CH}_{4}$ into CHA.[93] While not an exhaustive list, these studies and others in the literature point to a charge between 1.4 and 2.7 for the $\mathrm{Si}$ in a siliceous zeolite. This range spans the values produced by REPEAT to those determined from DDEC and iterative Hirshfeld. The most common value that appears in the literature is $\mathrm{q}_{\mathrm{Si}} \approx 2.2$, lending credence to the charges determined by the DDEC, iterative Hirshfeld, or EQEq methods. However, it is difficult to rank any charge (which cannot be measured directly) as the "best", so the performance of the models in predicting measurable properties needs to be considered.

\subsection{The Relationship Between Acidic/Catalytic Sites and Partial Atomic Charges}

The determination of a zeolite's catalytic site experimentally has been a heavily investigated topic over the past few decades. A leading hypothesis is that the T-site with the largest T-O-T angle should be the acidic site going along with the concepts of strain in the framework and the ionic character of a metal-oxygen-metal bond increasing with an increased bond angle.[94] However, Sastre et al. (and others) have shown little to no direct 
correlation between local structure and the acidic site. Instead, they conjectured that the acidity arises from the long-range electrostatics.[95,96] In a different study, Gale showed the classical- and cluster-based models often mispredict the site of interest because of a neglect of long-range electrostatics and that full periodic supercell simulations are more likely to make correct predictions.[97] Contrarily, Yang et al. asserted that distortions away from a regular $\mathrm{TO}_{4}$ tetrahedron (in terms of the $\mathrm{RMS}$ deviation of the O-T-O angles at a site) are what cause a T-site to be more acidic.[58] As zeolites are acid catalysts, one hypothesis is that the catalytic site will be the most acidic and therefore have the most positive charge in the system from its Lewis acidity. An aluminosilicate has Brønsted acidity from the presence of a hydroxyl formed from the association of a $\mathrm{H}^{+}$to an oxygen bound to an $\mathrm{Al}$. It is reasonable to expect the $\mathrm{H}^{+}$to associate with the oxygen atom having the most negative charge and where neighboring T-atoms will likely bear a higher positive charge. As the charge determination methodologies readily predict the most positive site in a zeolite, so we tested this hypothesis by analyzing a few well-studied, commercially relevant zeolite frameworks: BEA, BEC, MFI, MWW, and TON. Using only siliceous frameworks, this will also determine if the acidic sites can be predicted a priori by treating each T-site equivalently from a parameterization/pseudo-potential point of view.

Table 3: A comparison of the partial charges determined for the DFT optimized structure of BEA. The site 'Label' is the one used in the IZA-SC database. Literature identified Tsites of interest are in grey.

\begin{tabular}{|l|llllllll|}
\hline \hline Label & $\boldsymbol{E E M}$ & $\boldsymbol{Q E q}$ & $\boldsymbol{E Q E q}$ & $\boldsymbol{Q T P I E}$ & $\boldsymbol{H I}$ & DDEC & Bader & REPEAT \\
\hline \hline T1 & 1.6045 & 1.2446 & 2.1724 & 0.0669 & 2.5908 & 3.6562 & 2.2824 & 1.3139 \\
$\mathrm{~T} 2$ & 1.6049 & 1.2378 & 2.1808 & 0.0669 & 2.5938 & 2.7429 & 2.9094 & 1.3151 \\
$\mathrm{~T} 3$ & 1.6003 & 1.2580 & 2.2366 & 0.0662 & 2.5812 & 2.5536 & 2.7367 & 1.2516 \\
$\mathrm{~T} 4$ & 1.5996 & 1.2566 & 2.2355 & 0.0664 & 2.5824 & 2.1657 & 2.9270 & 1.2395 \\
T5 & 1.6084 & 1.2351 & 2.3960 & 0.0782 & 2.6091 & 2.6920 & 2.9683 & 1.3201 \\
T6 & 1.5968 & 1.2379 & 2.3589 & 0.0778 & 2.5996 & 2.3176 & 3.1222 & 1.2508
\end{tabular}




\begin{tabular}{|l|llllllll|} 
T7 & 1.6179 & 1.2712 & 2.2849 & 0.0669 & 2.5945 & 2.8284 & 2.9523 & 1.2579 \\
T8 & 1.6136 & 1.2827 & 2.2673 & 0.0672 & 2.5890 & 1.9532 & 3.1693 & 1.1923 \\
T9 & 1.6131 & 1.2644 & 2.2770 & 0.0672 & 2.5928 & 2.7103 & 3.1828 & 1.3604 \\
\hline \hline
\end{tabular}

Since experimentally measured structures rely on data of variable quality (most are determined by powder X-ray diffraction) and the refinements use different approaches (especially with respect to the level of restraints/constraints), we were concerned that experimental structures would not be sufficiently self-consistent for our purposes. We have already analyzed the DLS76 structures, but these structures are highly idealized and can often be off from the experiment either in terms of unit cell volume or a specific structural feature. To determine if these small changes to the framework can have noticeable effect on the predicted partial atomic charges in the previous section, we re-optimized the DLS76 structures of our commercially relevant zeolite frameworks using periodic plane-wave DFT. Table 3 shows the partial atomic charges of each method for the T-sites in the optimized BEA structure (similar tables for the other structures are in the supplemental information). When compared to the values shown in Table 2, it can be readily seen that the charge for each site changed following the DFT optimization. However, the predicted magnitudes of the charges do not change significantly $(<0.3 e)$. The EQEq and Bader charges experienced the greatest change in magnitude with the average $\mathrm{T}$-site charges dropping to $2.267 \mathrm{e}$ and $2.916 \mathrm{e}$, respectively. On the other hand, the HI and REPEAT charges for the optimized structures are much closer $(<0.03 \mathrm{e})$ to the DLS76 structures' charges: $2.593 \mathrm{e}$ and $1.278 \mathrm{e}$ respectively. In addition to changing values at each site, the ordering of sites by charge magnitude also changed with optimization for each method. For example, the site T5 has the greatest REPEAT charge for the DLS76 structure, yet the site T9 has the greatest REPEAT charge for the DFT optimized structure. This demonstrates 
just how sensitive these charge determinations are to structure; upon optimization, there was only a slight $1.4 \%$ increase in volume. That volume increase arose chiefly from an elongation of the crystallographic $c$-axis, which had the consequence of increasing the eccentricity of the elliptical 12MR channels of BEA. Because of the sensitivity of the charges to structure, the comparison with the literature for determining acidic sites will be done with the higher quality DFT optimized structures.

The literature provides several different (often inconsistent) accounts of which site(s) are the most acidic or the most favorable for substitution for each zeolite; conclusions vary depending on how the problem was approached and the specific experimental conditions. Studies devoted to the determination of the acid site are less numerous than those that focus on determining the preferred $\mathrm{T}$-atom $(\mathrm{T}=\mathrm{Sn}, \mathrm{Ge}, \mathrm{Ti}$, etc) substitution site as the site that will act as the catalytic site post-substitution. It is worth noting that the IZA-SC and experimental structures frequently have different site labels. For consistency, we will use the IZA-SC site notation here. Extended X-ray adsorption fine structure spectroscopy and periodic DFT simulations comparing HOMO-LUMO gaps of substituted frameworks have identified the T5/T6 site of BEA as the primary substitution site, consistent with the large T-O-T angle between sites T5 and T6.[36,98] These studies also indicated that the T5/T6 site should be the most acidic. The later work of Yang et al. argues that, while $\mathrm{T} 6$ is the most favorable substitution site, $\mathrm{T} 9$ should be the most acidic site based on simulated water adsorption even though the least stable substitution site.[58] The T5 and T9 sites of BEA are illustrated in Figure 1; similar cartoons for the other materials are available in the supplemental information. For BEC (the pure polymorph $\mathrm{C}$ of BEA with higher crystallographic order), the primary substitution site for metal atoms was 
determined to be at $\mathrm{T} 1$ in the $\mathrm{t}-\mathrm{d} 4 \mathrm{r}$ unit. However, $\mathrm{T} 3$ was shown to become the most favorable with increasing Ge concentration.[99-101] The description of MFI is the most varied. A recent review concluded that most sites were identified as a preferred substitution site by at least one study, but sites T8 and T10 are implicated most frequently.[102] Part of the difficulty may arise from the high flexibility in MFI. The MFI framework can adopt at least two distinct monoclinic and orthorhombic phases with heating or guest molecule insertion.[28,29,103-105] Later simulation studies have identified the acidic site as T11, T7, or T12.[106-109] Gale's study into MFI that expressed the need for correct-long range electrostatics identified T4 as the primary substitution site, which was corroborated with Ti and Fe substitution studies.[97,110-112] Hydroxyl IR studies ranked sites T1, T3, and T5 as the most acidic in MWW, with T6 as the most stable substitution site and T8 the least.[95] A later experimental and QM/MM study indicated that Al substitution prefers sites: $\mathrm{T} 6>\mathrm{T} 3>\mathrm{T} 8$, and the acidity went as $\mathrm{T} 8>\mathrm{T} 3>\mathrm{T} 6$ in the presence of acetone and T6 > T8 > T3 in the presence of TMPO.[96] Early cluster simulations indicate that $\mathrm{T} 1$ and $\mathrm{T} 4$ are the most favorable Al substitution sites in TON.[113] However, a later NMR and simulation study indicated T4 would be the most favorable and almost no substitution should occur at T1.[114] Early cluster simulations mark T2 as the most acidic site,[115] yet a later cluster study selected T1 as the most stable, accessible Brønsted acid site (although other sites it, they surpassed were dubbed inaccessible).[109] A more recent ab initio molecular dynamics study on the mechanism of propene methylation in TON identified T3 as the Brønsted acid site.[116]

The different charge determination methods provide differing descriptions of the site-to-site charge variations, and they each identify different sites as the most positive. 
EQEq, QTPIE, and HI all predict T5 and T6 as the first and second most positive sites in BEA, whereas Bader and REPEAT identify T9 as the most positive site. The charge difference between the first and second most positive sites in BEA is large enough ( $34 \%$ of the total for HI) to suggest chemically significant distinctions between the sites. Somewhat surprisingly, DDEC has a large distribution of charges (1.95e-3.65e) in BEA and the other zeolites, and it picks T1 as the most positive site in BEA. DDEC is the only method to predict site $\mathrm{T} 1$ as the most positive site in BEC, and only EEM, EQEq, HI, and REPEAT predict the other literature indicated site, T3, as the most positive site of BEC. The literature BEC studies used force field and cluster simulations, both of which were noted by Gale as prone to incorrect predictions.[97] That casts enough of a shadow of doubt to make assessment of quality difficult. REPEAT is the only method that predicts site $\mathrm{T} 4$ as the most acidic in MFI, following Gale's prediction. Considering the other methods, we see that Bader predicts T8 and DDEC T1 for MFI. HI, EQEq, and EEM all agree on site T3 for MFI, with REPEAT indicating that should be the second most acidic site. No method agreed with the substitution literature on MWW, placing T6 or T1 as the most positive site. QEq and REPEAT agreed with the QM/MM study identifying T8 as the most positive site, and DDEC was the sole method that selected T3 as the most acidic site. Most of the charge determination methods agree that site T4 is be the most positive site in TON, with DDEC as the only ab initio method to predict T1 as the most positive. The margins between the charges at different sites are the lowest for TON, making it seem that T2 and T4 should have comparable acidity according to Bader and HI.

Overall, the REPEAT method appears to yield the best agreement for the most electropositive T-site compared with the most reliable literature. An exception is the case of 
BEC, and to a lesser extent TON, where REPEAT agrees with most of the other methods. DDEC is the only $a b$ initio method that agrees with the literature determination of $\mathrm{T} 1 \mathrm{for}$ BEC and TON. Both these charge determination methods use the DFT periodic electrostatic potential (and thus long-range electrostatics) to determine the charges, so it is sensible they are the most adept at predicting acidic sites. HI also does a reasonable job of predicting the literature-identified substitution sites. A unique aspect of our approach compared to other investigations is that we used DFT-optimized structures while most other investigators performed simulations on the experimental structure. Considering the differences noted above, this is likely a major reason why we reach slightly different conclusions in some cases, especially compared with cluster and QM/MM studies. An additional point is that, in the optimization of MFI, we discovered two different solutions in the same range of unit cell volumes, and the lower energy solution reported more closely resembles the high temperature, evacuated phase of MFI.

To see if there is any connection between partial atomic charge for a T-site and its local structure, we analyzed the dependence on the local bond angles for the leading $a b$ initio charge determination methods. Following Yang's assertion, the T-sites with the greatest RMS deviation of O-T-O angles should be the most acidic,[58] and Figure 2 illustrates those deviations for the five zeolites using three different sets of charges. The average O-T-O angle in each case is the anticipated $\sim 109.47^{\circ}$. In each of the presented cases, the site with the largest RMS O-T-O deviation was not the site with the highest charge. The data show no clear correlation between predicted charge and RMS O-T-O deviation. The scatter evident in Figure 2 clearly shows that, for the most promising methods investigated here, O-T-O deviations have at best a secondary effect on partial 
charge. There is, however, some evidence of correlation between the T-O-T angles and predicted partial charges (Figure 3). Specifically, for the HI method, charges track the average T-O-T angles quite well, although not quantitatively. By its design, the HI method should do the best job of predicting the ionic character of a bond. Considering that more linear metal-oxygen-metal bond angles are expected to be more ionic, the observed correlation is expected. In TON, there are several linear T-O-T bonds in the system. We interpret that those large angles strain the tetrahedra, leading to the higher than average RMS deviations of the O-T-O and T-O-T angles. Since all these sites show higher simulated charges in HI, there is likely a relationship although far from perfect. For example, site T2 is connected via two linear T-O-T angles (giving the second highest average T-O-T angle in the cell), but the average O-T-O angle at $\mathrm{T} 2$ is the closest to the anticipated value with an RMSD of $1.73^{\circ}$.

The degree of correlation between RMS deviations of the T-O-T angles and partial charge is about as low as the correlation between the partial charges and the RMS O-T-O deviations, ie. the HI and DDEC plots just look like scatter plots. The REPEAT method appears to have a weak dependence on the RMS T-O-T deviations, but again it is not significant enough to be quantitative. We cannot exclude the possibility that stronger correlations might emerge when considering multiple, coupled geometric factors. However, as summarized at the start of this section, other teams have attempted to extract similar relationships from local geometry without success. Our results are consistent with their conclusions that long range correlations are at least as important as local geometry.

\section{3: The Effect of Compression on Partial Atomic Charges}


As shown in Tables 2 and 3, subtle changes in a zeolite's structure influence the predicted partial atomic charge. Compressing a zeolite under high pressure is one of the best physical ways to experimentally manipulate and change a zeolite's structure, and it has historically been one of the best tests for a forcefield's ability to accurately a zeolite framework. Previously Colligan et al. investigated the compression of FAU in both siliceous and cation containing forms with both synchrotron powder X-ray diffraction and force field structural optimizations.[80] The compression of siliceous FAU was probed with several pressure transmitting mediums including silicone oil. As silicone oil is too large to penetrate the interior of the zeolite, compression in it provides the hydrostatic compression of the framework under only external pressure. Diffraction data were collected until amorphization at $\mathrm{P} \geq 2.4 \mathrm{GPa}$. We used an equation of state extracted from their compression data to construct compressed structures at $0,0.5,1.0,1.5$ and $2.0 \mathrm{GPa}$. The respective lattice constants are 24.245, 24.142, 24.044, 23.9508, and $23.862 \AA$. As in-situ high pressure diffraction studies of powder samples do not typically provide sufficiently reliable atomic positions, we used DFT to refine the atomic coordinates (optimized structures in the SI). As was previously reported, the T-O lengths and O-T-O varied very little during compression, decreasing by at most $0.005 \AA$ and fluctuating between $0.002^{\circ}$ respectively. Adversely, the average T-O-T angle exhibited a large decrease as a function of pressure (Figure 4) indicating the twisting of the $\mathrm{TO}_{4}$ tetrahedra is the primary mechanism of deformation up to $2.0 \mathrm{GPa}$. Contrarily, the RMS deviation of the T-O-T angle increased with pressure (Figure 4). This is because, while the other T-O-T angles became more bent due to the compression, the T-O-T angle connecting the two 6MR of the d6r (t-hpr) composite building units become more linear. This matches the previously 
presented behavior predicted by empirical force fields reasonably well, and this differential distortion between the T-O-T angles involved in the sodalite cage ( $\beta$-cage, $\mathrm{t}$-sod) and the one purely involved in the d6r has been noted as the cause of amorphization in compressed FAU.[80,117] The RMS deviation of the T-O bond lengths and O-T-O angles also increased upon compression, although to a much smaller degree than the T-O-T angles: $0.003 \AA$ and $0.723^{\circ}$ respectively.

FAU is an opportune candidate to track the evolution of partial atomic charge with structural distortion as there is only crystallographically unique T-atom, which simplifies the analysis and allows for a clear representation of trends. Figure 5 shows the charges for the single crystallographically unique $\mathrm{Si}$ atom of each of the four optimized FAU structures predicted by the different ab initio methods. The predicted charges have magnitudes in agreement with those found for other the other zeolite structures with Bader, DDEC and HI between $2.5 \mathrm{e}$ and 3.0e, and the REPEAT charges significantly smaller around 1.1e. The HI and REPEAT charges exhibit a monotonic decrease in charge on the Si atom with increased pressure. The decrease is small, but it demonstrates a change in the chemical environment about the Si upon compression that a force field should reflect. The Bader charges also exhibit a decrease with increased pressure save a spike up at 2.0 GPa, and the DDEC charges exhibit two distinct sets of values $\geq 0.5 \mathrm{GPa}$ and between 1.0 and 2.0 GPa. This errant behavior of Bader and DDEC is somewhat expected as the atoms-in-molecules parsing will be the most susceptible to any errors like the equation of state fit or convergence criterion. As expected, the HI charges are the most consistent with the smallest variation between the different compressed structures, indicating that the valence state and ionic character of the Si-O bonds do not change with this amount of compression. 
Although, the general downward trend with respect to pressure does indicate an increased covalent character. Similarly, when going from the large-pore to the narrow-pore structure of a flexible MOF only a minor variation of the atomic charges on the chains is observed, also indicating the valence does not change.[118] The variation in covalent nature may be seen when you change the $+\mathrm{U}$ value for $\mathrm{DFT}+\mathrm{U}$ (this is more artificial as $+\mathrm{U}$ drives electron localization onto the target atoms)[119], which gives a steady increase of the Ti charges in the COK-69 MOF.[120] Previous studies have shown that, for inorganic compounds, lower metal-oxygen-metal bending angles tend to be more covalent.[121,122] The changes in the Si charges predicted by REPEAT closely track the changes in the volume the cell during compression. We interpret this to mean that the electrostatic potential is scaling relative to the change in the size of the void volumes. As observed in the previous sections, the decrease in $\mathrm{HI}$ and REPEAT charges with respect to pressure follows the decrease in the average T-O-T angles. These results also refute the assertion of Yang et al.,[58] since, as pressure increases, the HI and REPEAT charges decrease despite the RMS deviation of each feature (including O-T-O angles) increasing as a function of pressure.

\subsection{Performance in Predicting Gas Adsorption}

One of the most common applications of partial atomic charges is to perform force field gas simulations to model gas adsorption capacity, the heat of adsorption, and a material's ability to separate gases. We have previously studied the ability of the siliceous zeolite frameworks to separate $\mathrm{Kr}$ and $\mathrm{Xe}$ gas mixtures.[27] $\mathrm{Kr}$ and $\mathrm{Xe}$ have no partial atomic charges and cannot be used to test the applicability of our determined partial charges, so $\mathrm{N}_{2}$ and $\mathrm{O}_{2}$ which have partial atomic charges to replicate their standing 
molecular quadrupole moments are evaluated here. The adsorption of $\mathrm{N}_{2}$ and $\mathrm{O}_{2}$ into the FAU framework was simulated with GCMC using the same TraPPE-zeo dispersionrepulsion parameters employed in our previous study. We then compare these simulations experimental adsorption data measured in-house. The structure fully optimized with DFT (as in Section 3.2) and with charges determined from the ab initio methods was used. The partial charges on the one crystallographically unique tetrahedral atom of FAU are $1.140<$ $1.500<2.357<2.556<2.953$ for REPEAT, TraPPE-zeo (force field, denoted TZ), HI, DDEC, and Bader methods respectively. As may be seen in Figure 6, the different partial charges do make a difference in predicted adsorption for both the isotherm and predicted heat of adsorption. The simulated isotherms and heats of adsorption increase with higher partial charges on the T-site with the differences in magnitude (roughly) proportional to the differences in partial charge. The large difference in partial charge between REPEAT / TraPPE-zeo and the other methods is quite apparent in the simulated $\mathrm{N}_{2}$ adsorption; a similar split is also present for $\mathrm{O}_{2}$ but with a much smaller magnitude due primarily to oxygen's much smaller quadrupole moment (and partial atomic charges).

The close agreement between the REPEAT and TraPPE-zeo charges is logical because REPEAT is a method that fits charges to the DFT electrostatic potential (see the fuller discussion of the methods in the SI). Charges fit to the electrostatic potential should describe the electrostatic fields felt by guest molecules within a porous material well, like directly fitting charges to adsorption data. The atoms-in-molecules methods that atomistically partition the total DFT charge density (Bader and Hirshfeld) are known to produce charges that can incorrectly reproduce the electrostatic potential of the system.[46] Therefore, it is not surprising that the Bader charges produce the worst agreement with the 
experimental data, particularly at higher loadings. DDEC is a modification of Bader partitioning which should improve agreement with respect to the electrostatic potential in the pores of a material, and it does indeed improve the description of the adsorption properties of the Bader charges. The Hirshfeld and DDEC charges produce very similar adsorption properties (their $\mathrm{N}_{2}$ isotherms are almost on top of each other), and they produce the best agreement at low loadings. This could be an indication that they are both coming close to correctly reproducing the electrostatic potential at the internal surface of the zeolite.

The most eye-catching detail is how most of these combinations, including the defined charges for TraPPE-zeo, under-predicts the experimental adsorption at low loadings. In our previous investigations, we noted that just a 12-6 Lennard-Jones dispersion-repulsion for the adsorbate species did lead to an under-prediction, especially at high loadings for relatively low temperatures near the critical temperature in large pore materials like HKUST-1 and FAU.[123] The remedy was to utilize a 9-6 Lennard-Jones potential that was parameterized for the liquid state for the fluid-fluid interactions.[124] Even then, the predicted isotherms for $\mathrm{Kr}$ and $\mathrm{Xe}$ into a sample of FAU from the same batch under-predicted experimental adsorption by $5-15 \%$ at various temperatures. All that considered, the HI and DDEC charges are the best at reproducing the experimental adsorption at low loadings, and the TraPPE-zeo charges are the best at higher loadings. However, it is unreasonable to conclude that a simple substitution of charges into an existing force field would produce perfect agreement because many common zeolite force fields are fit for different purposes and with their own respective charge models as is evident by the TraPPE-zeo charges performing the best at higher loadings. To further 
demonstrate this, we performed additional GCMC simulations combining the iterative Hirshfeld charges with the dispersion-repulsion parameters of several other common force fields (Figures in SI). As expected, there was no significant improvement in agreement. A better next generation force field for simulating zeolites could be constructed by refining the dispersion-repulsion and bonding parameters for the zeolite framework with the better determined partial atomic charges produced by the iterative Hirshfeld or the similarly valued EQEq method.

To gauge whether the site-to-site variations in charges mattered for predicting adsorption compared to a one-charge fits all type approach, we created a hypothetical set of partial atomic charges that featured site-to-site variations yet had the same average values as the base TraPPE-zeo charges which uses only a single charge for Si and O. The REPEAT charges are the closest in value to the TraPPE-zeo charges, so they were scaled so that their average values matched the TraPPE-zeo charges. While FAU, with its fairly uniform internal surface (compared to other zeolites like BEA and MFI) may not be the best example to show the differences from using site-specific charges, Figure 7 clearly shows a difference in the GCMC predicted adsorption for $\mathrm{N}_{2}$ and $\mathrm{O}_{2}$ using the REPEAT, scaled REPEAT, and TraPPE-zeo charges. Increasing the magnitude of the REPEAT charges did increase the overall predicted adsorption, but it is still not equivalent to the adsorption predicted using the single valued TraPPE-zeo charges. The scaled REPEAT charges do exhibit a smaller predicted adsorption than the TraPPE-zeo charges, and this is most likely because the oxygen atoms in the adsorption site at the 6MR window into the $\beta$ cage have lower than average charges $(-0.728 \mathrm{e}$ and $-0.686 \mathrm{e})$ compared to the other oxygen atoms (-0.768e and -0.817e). This inhomogeneity in charge clearly leads to a lower 
electrostatic potential at the solid-fluid interface in FAU compared to just using a fixed 0.75 e for each oxygen, which in turn directly affects the predicted adsorption.

The adsorption energy of a single molecule into a framework removes the molecule-molecule interactions present in a full isotherm, allowing a direct probe of the effect of changing the partial atomic charges on adsorption simulations. The trends in adsorption energy shown in Table 4 follow those of the isotherms in Figure 6: the REPEAT and TraPPE-zeo charges are similar and the lowest adsorption energies, the iterative Hirshfeld and DDEC charges produce similar energetics and are in the middle, and the Bader charges give the highest energetics. The $\sim 33 \%$ larger TraPPE-zeo charges results in Coulombic contributions to the energy that are twice that of the REPEAT charges for $\mathrm{N}_{2}$, $\mathrm{O}_{2}, \mathrm{CO}_{2}, 2.3$ times larger for benzene, and 2.7 times larger for the dipolar gases $\left(\mathrm{H}_{2} \mathrm{O}\right.$ and $\mathrm{NH}_{3}$ ). The Bader charges which are $~ 2.6$ times larger than the REPEAT charges provide a Coulombic contribution to the interaction energy that is 8-10 times larger for the quadrupolar gases and 13-15 times larger for the dipolar gases, providing a litmus for the sensitivity of simulated adsorption properties to the chosen partial atomic charges. There was little effect on the adsorption energy for $\mathrm{O}_{2}$ from changing the zeolite's partial atomic charges owing to it having the smallest multipole of any of the gases in Table 4. On the other hand, benzene's Coulombic interaction energies range from 1.26 to $10.95 \mathrm{~kJ} / \mathrm{mol}$, i.e. 4 to $27 \%$ of the total adsorption energy. The dipolar gases are even more sensitive to the choice of framework partial atomic charges. The DDEC, iterative Hirshfeld, and Bader charges produce Coulombic contributions that are most of the total adsorption energy. They also show a marked decrease in the non-Coulombic energy $(1-6 \mathrm{~kJ} / \mathrm{mol})$, indicating that the 
adsorbed gas moved closer to the zeolite surface to maximize the total interaction at the cost of increasing the repulsive interaction.

In general, the predicted adsorption energies are too low, and this may be from a mismatch of the experimental structure and the one from the IZA-SC's database. However, the data for $\mathrm{N}_{2}$ and $\mathrm{O}_{2}$ in Figure 6 shows a quick drop off at low loading to values that better correspond to the adsorption energies produced with the iterative Hirschfeld and DDEC charges. The $\mathrm{H}_{2} \mathrm{O}$ study was simulation based and they claimed their predicted adsorption was too low but that included comparison to cases with a non-trivial amount of extra-framework cations.[125] The $\mathrm{NH}_{3}$ study also had some alumina-content in the framework $(\mathrm{Si} / \mathrm{Al}=10.29)$, but they showed a trend of increasing adsorption energy with decreased alumina content in H-substituted FAU that was plateauing at their highest measured $\mathrm{Si} / \mathrm{Al}$ ratio (the one shown here).[126] $\mathrm{NH}_{3}$ and $\mathrm{CO}_{2}$ are the cases with the best agreement with experiment. In those cases, the iterative Hirshfeld and DDEC charges provide the closest agreement, indicating they could be a good foundation for building future force fields.

Table 4: Several small (rigid) molecule adsorption energies (kJ/mol) into siliceous FAU computed with the different $a b$ initio framework partial atomic charges. Experimental heats of adsorption from: ${ }^{a}$ This work ${ }^{b}$ Ref. [127] ${ }^{\mathrm{c} S i m u l a t e d, ~ R e f . ~[125] ~}{ }^{\mathrm{d}} \mathrm{H}-\mathrm{FAU}$ (Si/Al=10.29) Ref. [126] ${ }^{\mathrm{e}}$ Ref. [128]

\begin{tabular}{|c|c|ccccc|}
\hline \hline Molecule & Expt. $\boldsymbol{\Delta H}^{\boldsymbol{0}}$ & $\boldsymbol{T Z}$ & $\boldsymbol{H I}$ & DDEC & Bader & REPEAT \\
\hline \hline $\mathrm{O}_{2} 140 \mathrm{~K}$ & $11.3^{\mathrm{a}}$ & 7.17 & 7.27 & 7.29 & 7.35 & 7.14 \\
$\mathrm{~N}_{2} 140 \mathrm{~K}$ & $12.0^{\mathrm{a}}$ & 7.51 & 8.53 & 8.35 & 9.17 & 7.23 \\
$\mathrm{~N}_{2} 300 \mathrm{~K}$ & $11.7^{\mathrm{b}}$ & 6.38 & 6.74 & 6.77 & 7.01 & 6.26 \\
$\mathrm{CO}_{2} 300 \mathrm{~K}$ & $16.8^{\mathrm{b}}$ & 11.97 & 15.30 & 15.44 & 17.04 & 10.98 \\
$\mathrm{H}_{2} \mathrm{O} 335 \mathrm{~K}$ & $17.0^{\mathrm{c}}$ & 11.16 & 29.62 & 24.05 & 39.02 & 6.85 \\
$\mathrm{NH}_{3} 298 \mathrm{~K}$ & $27.2^{\mathrm{d}}$ & 13.62 & 26.07 & 20.31 & 32.26 & 10.22 \\
$\mathrm{C}_{6} \mathrm{H}_{6} 300 \mathrm{~K}$ & $55.0^{\mathrm{e}}$ & 31.24 & 37.26 & 37.32 & 40.44 & 29.01 \\
\hline \hline
\end{tabular}

\section{$\underline{4 \text { Conclusions }}$}


We have shown that the different methodologies to compute partial atomic charges produce significantly different solutions when applied to the known pure silica zeolites. Atoms-in-molecule partitioning of the DFT charge density predicted the system to be the most ionic charges whereas all the other different methodologies predicted charges that better reflected the known covalency of the Si-O bond. All the methodologies showed at least some difference in charge between the different crystallographically unique sites with the RMS differences of the Bader charges being the least pronounced of all the methods. That these differences are physical and meaningful is evident from inspection of the zeolites' internal geometry where the chemical environment around each T-site is unique. Partial atomic charge analysis on the full periodic structure is a simple way to a priori predict the most catalytically active sites or sites most likely to undergo substitution in zeotype frameworks, especially since none of the simple local geometric metrics from the literature were found to correlate with the partial atomic charges. The closest connection to local geometry and partial atomic charge is the correlation between the average T-O-T angle at a T-site and the iterative Hirshfeld charges, but it is too weak to build a simplified, analytic algebraic model. Slight changes in the zeolite structure through structural optimization or compression experiments also had a noticeable impact on the predicted partial atomic charges and their variation between crystallographically unique atomic sites. This serves to emphasize the need for high quality, reliable structures for zeolite simulations.

Framework-to-framework and site-to-site variations in partial charge represent subtle, yet possibly important, features that will not be captured by fixed charge force fields, although they may be accounted for by core-shell potentials to at least some extent. 
While most current, reliable force fields can simulate properties like adsorption to within 5$10 \%$ of experiment, using site-specific partial atomic charges maybe a way to further improve agreement in future force field development, especially if that force field is to account for both framework flexibility and guest-host interactions. While the choice of partial atomic charges does have an impact on the prediction of gas adsorption, merely substituting 'better' partial atomic charges is not a sufficient solution. In many cases the van der Waals parameters are co-fit with the partial atomic charges, and like in Figure 7 substituting alternative charges could worsen the agreement with experiment. The solution would be to decide on the "best" partial atomic charges and then fit the other bonding and non-bonding force field parameters around them.

The lingering question is which choice of partial atomic charges is the most appropriate appears to depend on the specifics of the problem to be addressed. The iterative Hirshfeld charges described the strongly polar covalent nature of the bonding within the framework and tended to better predict substitution sites, whereas the REPEAT charges were better overall at predicting the acidic/catalytic site within a zeolite. Given their agreement with ab initio methods in both magnitude and variance between $\mathrm{T}$-sites, the $\mathrm{QEq} / \mathrm{EQEq}$ methods are likely to prove the best overall approach to predicting partial atomic charges once they have been tweaked to better reproduce the values and site-to-site variations of the REPEAT/iterative Hirshfeld charges. This is especially true for molecular dynamics where the charges should be recomputed on-the-fly to reflect the new bonding environment and where full $a b$ initio methods are prohibitively computationally expensive. The EQEq approach is likely better for zeolites because centering the Taylor expansion on the fully ionic states returns partial charges closely resembling the HI charges. For flexible 
framework simulations with zeolites, a better force field of sufficiently high quality that can describe the zeolite structure and interaction with guest species needs to be parameterized around the iterative Hirshfeld or tweaked EQEq charges.

\section{$\underline{\text { Acknowledgements }}$}

This research was sponsored (or sponsored in part) by the National Nuclear Security Administration under the Stewardship Science Academic Alliances program through DOE Cooperative Agreement \#DE-NA0001982. DEPV is a postdoctoral researcher funded by the Foundation of Scientific Research-Flanders (FWO) project no.12S3415N.

\section{$\underline{\text { References }}$}

[1] S.M. Csicsery, Shape-selective catalysis in zeolites, Zeolites. 4 (1984) 202-213. doi:10.1016/0144-2449(84)90024-1.

[2] M. Mabilia, R.A. Pearlstein, A.J. Hopfinger, Molecular modeling of zeolite structure. 1. Properties of the sodalite cage, J. Am. Chem. Soc. 109 (1987) 79607968. doi:10.1021/ja00260a005.

[3] Z.B. Bao, L. Yu, T. Dou, Y.J. Gong, Q. Zhang, Q.L. Ren, X.Y. Lu, S.G. Deng, Adsorption Equilibria of CO2, CH4, N-2, O-2, and Ar on High Silica Zeolites, J. Chem. Eng. Data. 56 (2011) 4017-4023. doi:10.1021/je200394p.

[4] A.F. Combariza, D.A. Gomez, G. Sastre, Simulating the properties of small pore silicazeolites using interatomic potentials, Chem. Soc. Rev. 42 (2013) 114-127. doi:10.1039/C2CS35243E.

[5] C.O. Arean, M.R. Delgado, P. Nachtigall, H.V. Thang, M. Rubeš, R. Bulánek, P. Chlubná-Eliášová, Measuring the Brønsted acid strength of zeolites - does it correlate with the $\mathrm{O}-\mathrm{H}$ frequency shift probed by a weak base?, Phys. Chem. Chem. Phys. 16 (2014) 10129-10141. doi:10.1039/C3CP54738H.

[6] M.E. Davis, New vistas in zeolite and molecular sieve catalysis, Acc. Chem. Res. 26 (1993) 111-115. doi:10.1021/ar00027a006.

[7] I. Papai, A. Goursot, F. Fajula, D. Plee, J. Weber, Modeling of N2 and O2 Adsorption in Zeolites, J. Phys. Chem. 99 (1995) 12925-12932. doi:10.1021/j100034a035.

[8] C. Mellot, J. Lignieres, Monte Carlo Simulations of N 2 and O 2 Adsorption in Silicalite and CaLSX Zeolites, Mol. Simul. 18 (1997) 349-365. doi:10.1080/08927029708024130.

[9] A. Zecchina, C. Lamberti, S. Bordiga, Surface acidity and basicity: General concepts, Catal. Today. 41 (1998) 169-177. doi:10.1016/S0920-5861(98)00047-9. 
[10] L. Yang, K. Trafford, O. Kresnawahjuesa, J. Šepa, R.J. Gorte, D. White, An Examination of Confinement Effects in High-Silica Zeolites, J. Phys. Chem. B. 105 (2001) 1935-1942. doi:10.1021/jp002964i.

[11] R. V. Siriwardane, M.S. Shen, E.P. Fisher, Adsorption of CO2, N2, and O2 on natural zeolites, Energy and Fuels. 17 (2003) 571-576. doi:10.1021/ef0201351.

[12] S. van Donk, A.H. Janssen, J.H. Bitter, K.P. de Jong, Generation, characterization, and impact of mesopores in zeolite catalysts, Catal. Rev. Eng. 45 (2003) 297-319. doi:10.1081/cr-120023908.

[13] R.S. Pillai, S.A. Peter, R. V Jasra, Correlation of sorption Behavior of nitrogen, oxygen, and argon with Ca2+ locations in zeolite A: A grand canonical Monte Carlo simulation study, Langmuir. 23 (2007) 8899-8908. doi:10.1021/la700821n.

[14] C. Baerlocher, L.B. McCusker, Database of Zeolite Structures, (n.d.). http://www.iza-structure.org/databases/.

[15] M.J. Sanders, M. Leslie, C.R.A. Catlow, Interatomic potentials for SiO2, J. Chem. Soc. Chem. Commun. (1984) 1271. doi:10.1039/c39840001271.

[16] R.A. Jackson, C.R.A. Catlow, Computer Simulation Studies of Zeolite Structure, Mol. Simul. 1 (1988) 207-224. doi:10.1080/08927028808080944.

[17] B.G. Dick, A.W. Overhauser, Theory of the Dielectric Constants of Alkali Halide Crystals, Phys. Rev. 112 (1958) 90-103. doi:10.1103/PhysRev.112.90.

[18] J.D. Gale, Analytical Free Energy Minimization of Silica Polymorphs, J. Phys. Chem. B. 102 (1998) 5423-5431. doi:10.1021/jp980396p.

[19] S.L. Mayo, B.D. Olafson, W.A. Goddard, DREIDING: a generic force field for molecular simulations, J. Phys. Chem. 94 (1990) 8897-8909. doi:10.1021/j100389a010.

[20] J.B. Nicholas, A.J. Hopfinger, F.R. Trouw, L.E. Iton, Molecular modeling of zeolite structure. 2. Structure and dynamics of silica sodalite and silicate force field, J. Am. Chem. Soc. 113 (1991) 4792-4800. doi:10.1021/ja00013a012.

[21] A.K. Rappe, C.J. Casewit, K.S. Colwell, W.A. Goddard, W.M. Skiff, UFF, a full periodic table force field for molecular mechanics and molecular dynamics simulations, J. Am. Chem. Soc. 114 (1992) 10024-10035. doi:10.1021/ja00051a040.

[22] S. Buttefey, A. Boutin, C. Mellot-Draznieks, A.H. Fuchs, A simple model for predicting the $\mathrm{Na}+$ distribution in anhydrous $\mathrm{NaY}$ and $\mathrm{NaX}$ zeolites, J. Phys. Chem. B. 105 (2001) 9569-9575. doi:10.1021/jp0105903.

[23] R.T. Cygan, J.-J. Liang, A.G. Kalinichev, Molecular Models of Hydroxide, Oxyhydroxide, and Clay Phases and the Development of a General Force Field, J. Phys. Chem. B. 108 (2004) 1255-1266. doi:10.1021/jp0363287.

[24] Y.G. Bushuev, G. Sastre, Atomistic Simulations of Structural Defects and Water Occluded in SSZ-74 Zeolite, J. Phys. Chem. C. 113 (2009) 10877-10886. doi:10.1021/jp9013306.

[25] B. Liu, B. Smit, F. Rey, S. Valencia, S. Calero, A New United Atom Force Field for Adsorption of Alkenes in Zeolites, J. Phys. Chem. C. 112 (2008) 2492-2498. doi:10.1021/jp075809d.

[26] P. Bai, M. Tsapatsis, J.I. Siepmann, TraPPE-zeo: Transferable potentials for phase equilibria force field for all-silica zeolites, J. Phys. Chem. C. 117 (2013) 2437524387. doi:10.1021/jp4074224.

[27] K. V. Lawler, A. Sharma, B. Alagappan, P.M. Forster, Assessing zeolite frameworks 
for noble gas separations through a joint experimental and computational approach, Microporous Mesoporous Mater. 222 (2016) 104-112.

doi:10.1016/j.micromeso.2015.10.005.

[28] H. van Koningsveld, J. Jansen, H. van Bekkum, The monoclinic framework structure of zeolite H-ZSM-5. Comparison with the orthorhombic framework of as-

synthesized ZSM-5, Zeolites. 10 (1990) 235-242. doi:10.1016/0144-2449(94)901341.

[29] H. van Koningsveld, J.C. Jansen, H. van Bekkum, The location of p-dichlorobenzene in a single crystal of zeolite H-ZSM-5 at high sorbate loading, Acta Crystallogr. Sect. B Struct. Sci. 52 (1996) 140-144. doi:10.1107/S0108768195008524.

[30] J.B. Parra, C.O. Ania, D. Dubbeldam, T.J.H. Vlugt, J.M. Castillo, P.J. Merkling, S. Calero, Unraveling the Argon Adsorption Processes in MFI-Type Zeolite, J. Phys. Chem. C. 112 (2008) 9976-9979. doi:10.1021/jp803753h.

[31] S. Chempath, L.A. Clark, R.Q. Snurr, Two general methods for grand canonical ensemble simulation of molecules with internal flexibility, J. Chem. Phys. 118 (2003) 7635-7643. doi:doi:http://dx.doi.org/10.1063/1.1562607.

[32] D. Dubbeldam, R.Q. Snurr, Recent developments in the molecular modeling of diffusion in nanoporous materials, Mol. Simul. 33 (2007) 305-325. doi:10.1080/08927020601156418.

[33] Y.G. Bushuev, G. Sastre, Feasibility of Pure Silica Zeolites, J. Phys. Chem. C. 114 (2010) 19157-19168. doi:10.1021/jp107296e.

[34] D. Dubbeldam, S. Calero, D.E. Ellis, R.Q. Snurr, RASPA: molecular simulation software for adsorption and diffusion in flexible nanoporous materials, Mol. Simul. 42 (2016) 81-101. doi:10.1080/08927022.2015.1010082.

[35] J. Weitkamp, Zeolites and catalysis, Solid State Ionics. 131 (2000) 175-188. doi:10.1016/s0167-2738(00)00632-9.

[36] S.R. Bare, S.D. Kelly, W. Sinkler, J.J. Low, F.S. Modica, S. Valencia, A. Corma, L.T. Nemeth, Uniform Catalytic Site in Sn- $\beta$-Zeolite Determined Using X-ray Absorption Fine Structure, J. Am. Chem. Soc. 127 (2005) 12924-12932. doi:10.1021/ja052543k.

[37] W.O. Haag, CATALYSIS BY ZEOLITES - SCIENCE AND TECHNOLOGY, in: J. Weitkamp, H.G. Karge, H. Pfeifer, W. Holderich (Eds.), Zeolites Relat. Microporous Mater. State Art 1994, Elsevier Science Bv, Amsterdam, 1994: pp. 1375-1394.

[38] S. Hamad, S.R.G. Balestra, R. Bueno-Perez, S. Calero, A.R. Ruiz-Salvador, Atomic charges for modeling metal-organic frameworks: Why and how, J. Solid State Chem. 223 (2015) 144-151. doi:10.1016/j.jssc.2014.08.004.

[39] W.J. Mortier, S.K. Ghosh, S. Shankar, Electronegativity-equalization method for the calculation of atomic charges in molecules, J. Am. Chem. Soc. 108 (1986) 43154320. doi:10.1021/ja00275a013.

[40] A.K. Rappe, W.A. Goddard, Charge equilibration for molecular dynamics simulations, J. Phys. Chem. 95 (1991) 3358-3363. doi:10.1021/j100161a070.

[41] C.E. Wilmer, K.C. Kim, R.Q. Snurr, An Extended Charge Equilibration Method, J. Phys. Chem. Lett. 3 (2012) 2506-2511. doi:10.1021/jz3008485.

[42] J.H. Chen, T.J. Martinez, QTPIE: Charge transfer with polarization current equalization. A fluctuating charge model with correct asymptotics, Chem. Phys. Lett. 438 (2007) 315-320. doi:10.1016/j.cplett.2007.02.065. 
[43] F.L. Hirshfeld, Bonded-atom fragments for describing molecular charge densities, Theor. Chim. Acta. 44 (1977) 129-138. doi:10.1007/BF00549096.

[44] D.E.P. Vanpoucke, P. Bultinck, I. Van Driessche, Extending Hirshfeld-I to bulk and periodic materials, J. Comput. Chem. 34 (2013) 405-417. doi:10.1002/jcc.23088.

[45] D.E.P. Vanpoucke, I. Van Driessche, P. Bultinck, Reply to 'comment on "extending Hirshfeld-I to bulk and periodic materials," J. Comput. Chem. 34 (2013) 422-427. doi:10.1002/jcc.23193.

[46] T.A. Manz, D.S. Sholl, Chemically Meaningful Atomic Charges That Reproduce the Electrostatic Potential in Periodic and Nonperiodic Materials, J. Chem. Theory Comput. 6 (2010) 2455-2468. doi:10.1021/ct100125x.

[47] T.A. Manz, D.S. Sholl, Improved Atoms-in-Molecule Charge Partitioning Functional for Simultaneously Reproducing the Electrostatic Potential and Chemical States in Periodic and Nonperiodic Materials, J. Chem. Theory Comput. 8 (2012) 2844-2867. doi:10.1021/ct3002199.

[48] N.G. Limas, T.A. Manz, Introducing DDEC6 atomic population analysis: part 2. Computed results for a wide range of periodic and nonperiodic materials, RSC Adv. 6 (2016) 45727-45747. doi:10.1039/C6RA05507A.

[49] E. Sanville, S.D. Kenny, R. Smith, G. Henkelman, Improved grid-based algorithm for Bader charge allocation, J. Comput. Chem. 28 (2007) 899-908. doi:10.1002/jcc.20575.

[50] W. Tang, E. Sanville, G. Henkelman, A grid-based Bader analysis algorithm without lattice bias, J. Physics-Condensed Matter. 21 (2009) 7. doi:10.1088/09538984/21/8/084204.

[51] M. Yu, D.R. Trinkle, Accurate and efficient algorithm for Bader charge integration, J. Chem. Phys. 134 (2011) 64111. doi:doi:http://dx.doi.org/10.1063/1.3553716.

[52] C. Campañá, B. Mussard, T.K. Woo, Electrostatic Potential Derived Atomic Charges for Periodic Systems Using a Modified Error Functional, J. Chem. Theory Comput. 5 (2009) 2866-2878. doi:10.1021/ct9003405.

[53] C. Baerlocher, A. Hepp, W.M. Meier, DLS-76 : a program for the simulation of crystal structures by geometric refinement, English, R, Institute of Crystallography and Petrography - ETH, Zürich, 1978. http://www.worldcat.org/title/dls-76-aprogram-for-the-simulation-of-crystal-structures-by-geometricrefinement/oclc/428159919\&referer=brief_results (accessed September 18, 2018).

[54] G. Sastre, J.D. Gale, ZeoTsites: a code for topological and crystallographic tetrahedral sites analysis in zeolites and zeotypes, Microporous Mesoporous Mater. 43 (2001) 27-40. doi:10.1016/S1387-1811(00)00344-9.

[55] G. Sastre, A. Corma, Rings and Strain in Pure Silica Zeolites, J. Phys. Chem. B. 110 (2006) 17949-17959. doi:10.1021/jp060505x.

[56] J.D. Gale, GULP: A computer program for the symmetry-adapted simulation of solids, J. Chem. Soc. Trans. 93 (1997) 629-637. doi:10.1039/a606455h.

[57] J.P. Perdew, K. Burke, M. Ernzerhof, Generalized Gradient Approximation Made Simple, Phys. Rev. Lett. 77 (1996) 3865-3868. doi:10.1103/PhysRevLett.77.3865.

[58] G. Yang, E.A. Pidko, E.J.M. Hensen, Structure, Stability, and Lewis Acidity of Mono and Double Ti, Zr, and Sn Framework Substitutions in BEA Zeolites: A Periodic Density Functional Theory Study, J. Phys. Chem. C. 117 (2013) 39763986. doi:10.1021/jp310433r. 
[59] S. Grimme, J. Antony, S. Ehrlich, H. Krieg, A consistent and accurate ab initio parametrization of density functional dispersion correction (DFT-D) for the 94 elements H-Pu, J. Chem. Phys. 132 (2010) 154104. doi:10.1063/1.3382344.

[60] P.E. Blöchl, Projector augmented-wave method, Phys. Rev. B. 50 (1994) 1795317979. doi:10.1103/PhysRevB.50.17953.

[61] G. Kresse, D. Joubert, From ultrasoft pseudopotentials to the projector augmentedwave method, Phys. Rev. B. 59 (1999) 1758-1775. doi:10.1103/PhysRevB.59.1758.

[62] P. Vinet, J.R. Smith, J. Ferrante, J.H. Rose, Temperature effects on the universal equation of state of solids, Phys. Rev. B. 35 (1987) 1945-1953.

doi:10.1103/PhysRevB.35.1945.

[63] P.E. Blöchl, O. Jepsen, O.K. Andersen, Improved tetrahedron method for Brillouinzone integrations, Phys. Rev. B. 49 (1994) 16223-16233.

doi:10.1103/PhysRevB.49.16223.

[64] W. Kohn, L.J. Sham, Self-Consistent Equations Including Exchange and Correlation Effects*, Phys. Rev. A. 140 (1965) 1133-1138.

[65] G. Henkelman, A. Arnaldsson, H. Jonsson, A fast and robust algorithm for Bader decomposition of charge density, Comput. Mater. Sci. 36 (2006) 354-360. doi:10.1016/j.commatsci.2005.04.010.

[66] N. Metropolis, A.W. Rosenbluth, M.N. Rosenbluth, A.H. Teller, E. Teller, Equation of state calculations by fast computing machines, J. Chem. Phys. 21 (1953) 10871092. doi:http://dx.doi.org/10.1063/1.1699114.

[67] D.-Y. Peng, D.B. Robinson, A New Two-Constant Equation of State, Ind. Eng. Chem. Fundam. 15 (1976) 59-64. doi:10.1021/i160057a011.

[68] M.P. Allen, D.J. Tildesley, Computer Simulation of Liquids, Clarendon Press; Oxford University Press, New York, 1987.

[69] D. Frenkel, B. Smit, Understanding Molecular Simulation: From Algorithms to Applications, 2nd ed., Academic Press, Elsevier, San Diego, 2002. http://portal.acm.org/citation.cfm?id=559571.

[70] A. Gupta, S. Chempath, M.J. Sanborn, L.A. Clark, R.Q. Snurr, Object-oriented Programming Paradigms for Molecular Modeling, Mol. Simul. 29 (2003) 29-46. doi:10.1080/0892702031000065719.

[71] D. Wolf, P. Keblinski, S.R. Phillpot, J. Eggebrecht, Exact method for the simulation of Coulombic systems by spherically truncated, pairwise $\mathrm{r}-1$ summation, J. Chem. Phys. 110 (1999) 8254-8282. doi:10.1063/1.478738.

[72] D. Olds, K. V. Lawler, A.A. Paecklar, J. Liu, K. Page, P.F. Peterson, P.M. Forster, J.R. Neilson, Capturing the Details of N 2 Adsorption in Zeolite X Using Stroboscopic Isotope Contrasted Neutron Total Scattering, Chem. Mater. 30 (2018) 296-302. doi:10.1021/acs.chemmater.7b04594.

[73] J.J. Potoff, J.I. Siepmann, Vapor-liquid equilibria of mixtures containing alkanes, carbon dioxide, and nitrogen, AIChE J. 47 (2001) 1676-1682.

doi:10.1002/aic.690470719.

[74] L. Zhang, J.I. Siepmann, Direct calculation of Henry's law constants from Gibbs ensemble Monte Carlo simulations: nitrogen, oxygen, carbon dioxide and methane in ethanol, Theor. Chem. Acc. 115 (2006) 391-397. doi:10.1007/s00214-005-0073-1.

[75] L. Zhang, J.I. Siepmann, Development of the trappe force field for ammonia, Collect. Czechoslov. Chem. Commun. 75 (2010) 577-591. 
doi:10.1135/cccc2009540.

[76] C.D. Wick, J.I. Siepmann, W.L. Klotz, M.R. Schure, Temperature effects on the retention of $\mathrm{n}$-alkanes and arenes in helium-squalane gas-liquid chromatography, $\mathrm{J}$. Chromatogr. A. 954 (2002) 181-190. doi:10.1016/S0021-9673(02)00171-1.

[77] W.L. Jorgensen, J. Chandrasekhar, J.D. Madura, R.W. Impey, M.L. Klein, Comparison of simple potential functions for simulating liquid water, J. Chem. Phys. 79 (1983) 926-935. doi:10.1063/1.445869.

[78] K.V. Lawler, P.M. Forster, Evaluating the Selectivity of Sorbents for Noble Gas Separations across a Range of Temperatures, Loadings, and Gas Compositions, Zeitschrift Fur Anorg. Und Allg. Chemie. 642 (2016). doi:10.1002/zaac.201600375.

[79] J.A. Hriljac, M.M. Eddy, A.K. Cheetham, J.A. Donohue, G.J. Ray, Powder Neutron Diffraction and 29Si MAS NMR Studies of Siliceous Zeolite-Y, J. Solid State Chem. 106 (1993) 66-72. doi:10.1006/jssc.1993.1265.

[80] M. Colligan, P.M. Forster, A.K. Cheetham, Y. Lee, T. Vogt, J.A. Hriljac, Synchrotron X-ray Powder Diffraction and Computational Investigation of Purely Siliceous Zeolite Y under Pressure, J. Am. Chem. Soc. 126 (2004) 12015-12022. doi:10.1021/ja048685g.

[81] J.A. Hriljac, High-pressure synchrotron X-ray powder diffraction studies of zeolites, Crystallogr. Rev. 12 (2006) 181-193. doi:10.1080/08893110600772032.

[82] F.C. Jentoft, Advances in Catalysis, Vol. 57, Elsevier Academic Press Inc, San Diego, 2014.

[83] J.D. Gale, A.L. Rohl, The General Utility Lattice Program (GULP), Mol. Simul. 29 (2003) 291-341. doi:10.1080/0892702031000104887.

[84] R.S. Mulliken, Electronic Population Analysis on LCAO-MO Molecular Wave Functions. I, J. Chem. Phys. 23 (1955) 1833-1840. doi:10.1063/1.1740588.

[85] E. Aprà, R. Dovesi, C. Freyria-Fava, C. Pisani, C. Roetti, V.R. Saunders, Ab initio Hartree-Fock modelling of zeolites: application to silico-chabazite, Model. Simul. Mater. Sci. Eng. 1 (1993) 297-306. doi:10.1088/0965-0393/1/3/004.

[86] E.H. Teunissen, A.P.J. Jansen, R.A. van Santen, R. Orlando, R. Dovesi, Adsorption energies of NH 3 and $\mathrm{NH}+4$ in zeolites corrected for the long-range electrostatic potential of the crystal, J. Chem. Phys. 101 (1994) 5865-5874. doi:10.1063/1.467303.

[87] C.M. Zicovich-Wilson, R. Dovesi, Titanium-Containing Zeolites. A Periodic ab Initio Hartree-Fock Characterization, J. Phys. Chem. B. 102 (1998) 1411-1417. doi:10.1021/jp972343y.

[88] C. Møller, M.S. Plesset, Note on an Approximation Treatment for Many-Electron Systems, Phys. Rev. 46 (1934) 618-622. doi:10.1103/PhysRev.46.618.

[89] K. de Boer, A.P.J. Jansen, R.A. van Santen, Ab initio approach to the development of interatomic potentials for the shell model of silica polymorphs, Chem. Phys. Lett. 223 (1994) 46-53. doi:10.1016/0009-2614(94)00406-4.

[90] A.D. Becke, Density-functional thermochemistry. III. The role of exact exchange, J. Chem. Phys. 98 (1993) 5648-5652. doi:10.1063/1.464913.

[91] A. Pedone, G. Malavasi, M.C. Menziani, U. Segre, F. Musso, M. Corno, B. Civalleri, P. Ugliengo, FFSiOH: a New Force Field for Silica Polymorphs and Their Hydroxylated Surfaces Based on Periodic B3LYP Calculations, Chem. Mater. 20 (2008) 2522-2531. doi:10.1021/cm703437y. 
[92] H. Fang, P. Kamakoti, J. Zang, S. Cundy, C. Paur, P.I. Ravikovitch, D.S. Sholl, Prediction of CO 2 Adsorption Properties in Zeolites Using Force Fields Derived from Periodic Dispersion-Corrected DFT Calculations, J. Phys. Chem. C. 116 (2012) 10692-10701. doi:10.1021/jp302433b.

[93] H. Fang, R. Awati, S.E. Boulfelfel, P.I. Ravikovitch, D.S. Sholl, First-PrinciplesDerived Force Fields for CH 4 Adsorption and Diffusion in Siliceous Zeolites, J. Phys. Chem. C. 122 (2018) 12880-12891. doi:10.1021/acs.jpcc.8b03267.

[94] D. Barthomeuf, Zeolite acidity dependence on structure and chemical environment. Correlations with catalysis, Mater. Chem. Phys. 17 (1987) 49-71. doi:10.1016/02540584(87)90048-4.

[95] G. Sastre, V. Fornes, A. Corma, Preferential Siting of Bridging Hydroxyls and Their Different Acid Strengths in the Two-Channel System of MCM-22 Zeolite, J. Phys. Chem. B. 104 (2000) 4349-4354. doi:10.1021/jp993636p.

[96] A. Zheng, L. Chen, J. Yang, M. Zhang, Y. Su, Y. Yue, C. Ye, F. Deng, Combined DFT Theoretical Calculation and Solid-State NMR Studies of Al Substitution and Acid Sites in Zeolite MCM-22, J. Phys. Chem. B. 109 (2005) 24273-24279. doi:10.1021/jp0527249.

[97] J.D. Gale, A periodic density functional study of the location of titanium within TS1, Solid State Sci. 8 (2006) 234-240. doi:10.1016/j.solidstatesciences.2006.02.011.

[98] S. Shetty, B.S. Kulkarni, D.G. Kanhere, A. Goursot, S. Pal, A Comparative Study of Structural, Acidic and Hydrophilic Properties of Sn-BEA with Ti-BEA Using Periodic Density Functional Theory, J. Phys. Chem. B. 112 (2008) 2573-2579. doi:10.1021/jp709846s.

[99] A. Corma, M.T. Navarro, F. Rey, J. Rius, S. Valencia, Pure polymorph C of zeolite beta synthesized by using framework isomorphous substitution as a structuredirecting mechanism, Angew. Chemie-International Ed. 40 (2001) 2277-+. doi:10.1002/1521-3773(20010618)40:12<2277::aid-anie2277>3.0.co;2-o.

[100] G. Sastre, J.A. Vidal-Moya, T. Blasco, J. Rius, J.L. Jorda, M.T. Navarro, F. Rey, A. Corma, Preferential location of Ge atoms in polymorph $\mathrm{C}$ of beta zeolite (ITQ-17) and their structure-directing effect: A computational, XRD, and NMR spectroscopic study, Angew. Chemie-International Ed. 41 (2002) 4722-4726. doi:10.1002/anie.200290028.

[101] B.D. Montejo-Valencia, M.C. Curet-Arana, DFT Study of the Lewis Acidities and Relative Hydrothermal Stabilities of BEC and BEA Zeolites Substituted with Ti, Sn, and Ge, J. Phys. Chem. C. 119 (2015) 4148-4157. doi:10.1021/jp512269s.

[102] L. Nemeth, S.R. Bare, Science and Technology of Framework Metal-Containing Zeotype Catalysts, in: C.J. Friederike (Ed.), Adv. Catal., Academic Press, 2014: pp. 1-97. doi:10.1016/B978-0-12-800127-1.00001-1.

[103] H. van Koningsveld, H. van Bekkum, J.C. Jansen, On the location and disorder of the tetrapropylammonium (TPA) ion in zeolite ZSM-5 with improved framework accuracy, Acta Crystallogr. Sect. B Struct. Sci. 43 (1987) 127-132. doi:10.1107/S0108768187098173.

[104] H. van Koningsveld, F. Tuinstra, H. van Bekkum, J.C. Jansen, The location of pxylene in a single crystal of zeolite H-ZSM-5 with a new, sorbate-induced, orthorhombic framework symmetry, Acta Crystallogr. Sect. B. 45 (1989) 423-431. doi:doi:10.1107/S0108768189004519. 
[105] H. van Koningsveld, High-temperature ( $350 \mathrm{~K}$ ) orthorhombic framework structure of zeolite H-ZSM-5, Acta Crystallogr. Sect. B Struct. Sci. 46 (1990) 731-735. doi:10.1107/S0108768190007522.

[106] H. V Brand, L.A. Curtiss, L.E. Iton, Ab initio molecular orbital cluster studies of the zeolite ZSM-5. 1. Proton affinities, J. Phys. Chem. 97 (1993) 12773-12782. doi:10.1021/j100151a024.

[107] S.A. Zygmunt, L.A. Curtiss, L.E. Iton, M.K. Erhardt, Computational Studies of Water Adsorption in the Zeolite H-ZSM-5, J. Phys. Chem. 100 (1996) 6663-6671. doi:10.1021/jp952913z.

[108] R.Z. Khaliullin, A.T. Bell, V.B. Kazansky, An Experimental and Density Functional Theory Study of the Interactions of CH4 with H-ZSM-5, J. Phys. Chem. A. 105 (2001) 10454-10461. doi:10.1021/jp012377c.

[109] A. Simperler, R.G. Bell, M.D. Foster, A.E. Gray, D.W. Lewis, M.W. Anderson, Probing the Acid Strength of Brønsted Acidic Zeolites with Acetonitrile: An Atomistic and Quantum Chemical Study, J. Phys. Chem. B. 108 (2004) 7152-7161. doi:10.1021/jp035673t.

[110] K. Sun, F. Fan, H. Xia, Z. Feng, W.-X. Li, C. Li, Framework Fe Ions in Fe-ZSM-5 Zeolite Studied by UV Resonance Raman Spectroscopy and Density Functional Theory Calculations, J. Phys. Chem. C. 112 (2008) 16036-16041. doi:10.1021/jp802955s.

[111] G. Pirc, J. Stare, Titanium Site Preference Problem in the TS-1 Zeolite Catalyst: A Periodic Hartree-Fock Study, Acta Chim. Slov. 55 (2008) 951-959.

[112] S.P. Yuan, H.Z. Si, A.P. Fu, T.S. Chu, F.H. Tian, Y.B. Duan, J.G. Wang, Location of $\mathrm{Si}$ Vacancies and $\mathrm{Ti}(\mathrm{OSi})(4)$ and $\mathrm{Ti}(\mathrm{OSi})(3) \mathrm{OH}$ Sites in the MFI Framework: A Large Cluster and Full Ab Initio Study, J. Phys. Chem. A. 115 (2011) 940-947. doi:10.1021/jp110977m.

[113] P.J. O'Malley, J. Dwyer, Ab-initio molecular orbital calculations on the siting of aluminium in the Theta-1 framework: Some general guidelines governing the site preferences of aluminium in zeolites, Zeolites. 8 (1988) 317-321. doi:10.1016/S0144-2449(88)80129-5.

[114] S. Sklenak, J. Dědeček, C. Li, F. Gao, B. Jansang, B. Boekfa, B. Wichterlová, J. Sauer, Aluminum Siting in the ZSM-22 and Theta-1 Zeolites Revisited: A QM/MM Study, Collect. Czechoslov. Chem. Commun. 73 (2008) 909-920. doi:10.1135/ccce20080909.

[115] M. Boronat, C.M. Zicovich-Wilson, P. Viruela, A. Corma, Cluster and Periodic Calculations of the Ethene Protonation Reaction Catalyzed by theta-1 Zeolite: Influence of Method, Model Size, and Structural Constraints, Chem. Eur. J. 7 (2001) 1295-1303. doi:10.1002/1521-3765(20010316)7:6<1295::AIDCHEM1295>3.0.CO;2-S.

[116] K. De Wispelaere, S. Bailleul, V. Van Speybroeck, Towards molecular control of elementary reactions in zeolite catalysis by advanced molecular simulations mimicking operating conditions, Catal. Sci. Technol. 6 (2016) 2686-2705. doi: $10.1039 / \mathrm{c} 5$ cy02073e.

[117] E.A. Havenga, Y. Huang, R.A. Secco, An investigation of the effect of high pressure on the structure of siliceous zeolite Y, Mater. Res. Bull. 38 (2003) 381-387. doi:10.1016/S0025-5408(02)01061-9. 
[118] D.E.P. Vanpoucke, K. Lejaeghere, V. Van Speybroeck, M. Waroquier, A. Ghysels, Mechanical Properties from Periodic Plane Wave Quantum Mechanical Codes: The Challenge of the Flexible Nanoporous MIL-47(V) Framework, J. Phys. Chem. C. 119 (2015). doi:10.1021/acs.jpcc.5b06809.

[119] S.L. Dudarev, G.A. Botton, S.Y. Savrasov, C.J. Humphreys, A.P. Sutton, Electronenergy-loss spectra and the structural stability of nickel oxide: An LSDA+U study, Phys. Rev. B. 57 (1998) 1505-1509. doi:10.1103/PhysRevB.57.1505.

[120] B. Bueken, F. Vermoortele, D.E.P. Vanpoucke, H. Reinsch, C.-C. Tsou, P. Valvekens, T. De Baerdemaeker, R. Ameloot, C.E.A. Kirschhock, V. Van Speybroeck, J.M. Mayer, D. De Vos, A Flexible Photoactive Titanium MetalOrganic Framework Based on a [Ti IV $3(\mu 3-\mathrm{O})(\mathrm{O}) 2$ (COO) 6 ] Cluster, Angew. Chemie Int. Ed. 54 (2015) 13912-13917. doi:10.1002/anie.201505512.

[121] D.A. Dixon, J.L. Gole, Description of the ground state electronic structures of Cu2O, Cu2S, Ag2O and Ag2S, Chem. Phys. Lett. 189 (1992) 390-394. doi:10.1016/00092614(92)85220-5.

[122] F. Weigend, R. Ahlrichs, Balanced basis sets of split valence, triple zeta valence and quadruple zeta valence quality for $\mathrm{H}$ to $\mathrm{Rn}$ : Design and assessment of accuracy, Phys. Chem. Chem. Phys. 7 (2005) 3297. doi:10.1039/b508541a.

[123] K. V. Lawler, Z. Hulvey, P.M. Forster, On the importance of a precise crystal structure for simulating gas adsorption in nanoporous materials, Phys. Chem. Chem. Phys. 17 (2015) 18904-18907. doi:10.1039/C5CP01544H.

[124] J. Yang, Y. Ren, A.M. Tian, H.A. Sun, COMPASS force field for 14 inorganic molecules, $\mathrm{He}, \mathrm{Ne}$, Ar, Kr, Xe, H-2, O-2, N-2, NO, CO, CO2, NO2, CS2, and SO2, in liquid phases, J. Phys. Chem. B. 104 (2000) 4951-4957. doi:10.1021/jp992913p.

[125] M. Fleys, R.W. Thompson, Monte Carlo Simulations of Water Adsorption Isotherms in Silicalite and Dealuminated Zeolite Y, J. Chem. Theory Comput. 1 (2005) 453458. doi:10.1021/ct049896e.

[126] K. Tsutsumi, H.Q. Koh, S. Hagiwara, H. Takahashi, Direct Measurement of Interaction Energy between Solids and Gases. I. Heat of Adsorption of Ammonia on Zeolite, Bull. Chem. Soc. Jpn. 48 (1975) 3576-3580. doi:10.1246/bcsj.48.3576.

[127] G. Maurin, R. Bell, B. Kuchta, T. Poyet, P. Llewellyn, Adsorption of Non Polar and Quadrupolar Gases in Siliceous Faujasite: Molecular Simulations and Experiments, Adsorption. 11 (2005) 331-336. doi:10.1007/s10450-005-5946-z.

[128] N.J. Henson, A.K. Cheetham, M. Stockenhuber, J.A. Lercher, Modelling aromatics in siliceous zeolites: a new forcefield from thermochemical studies, J. Chem. Soc. Faraday Trans. 94 (1998) 3759-3768. doi:10.1039/a806175k. 


\section{$\underline{\text { Figure Captions }}$}

Fig. 1: The crystal structure of BEA along [100]. Site T5 is blue, site T9 is green, oxygens are red, and the remaining T-sites are black.

Fig. 2: A comparison of the RMS deviation of the O-T-O angles to the HI, DDEC, and REPEAT charges for each crystallographically unique T-site in the 5 zeolites.

Fig. 3: A comparison of the average (top) and RMS deviation (bottom) of the T-O-T angles to the HI, DDEC, and REPEAT charges for each crystallographically unique T-site in the 5 zeolites.

Fig. 4: A comparison of the average T-O-T angles (red squares) and the RMS T-O-T deviation (blue circles) in FAU compressed up to 2.0 GPa.

Fig. 5: A comparison of the ab initio predicted partial atomic charges in FAU compressed up to 2.0 GPa: Bader (red), DDEC (blue), HI (green), REPEAT (orange).

Fig. 6: Simulated and experimental (Expt) $\mathrm{N}_{2}$ (top) and $\mathrm{O}_{2}$ (bottom) adsorption isotherms (left) and heats of adsorption (right) for FAU at 140K. The TraPPE-zeo dispersionrepulsion parameters were used in all simulations. The measured/simulated points are distinguished with a marker. The isotherms using the HI (blue) and DDEC (green) charges lie almost on top of each other.

Fig. 7: Simulated $\mathrm{N}_{2}$ (top) and $\mathrm{O}_{2}$ (bottom) adsorption isotherms (left) and heats of adsorption (right) for FAU at 140K using the TraPPE-zeo (TZ), REPEAT, and scaled REPEAT (SR) charges. The simulated points are distinguished with a marker. 
Figure 1

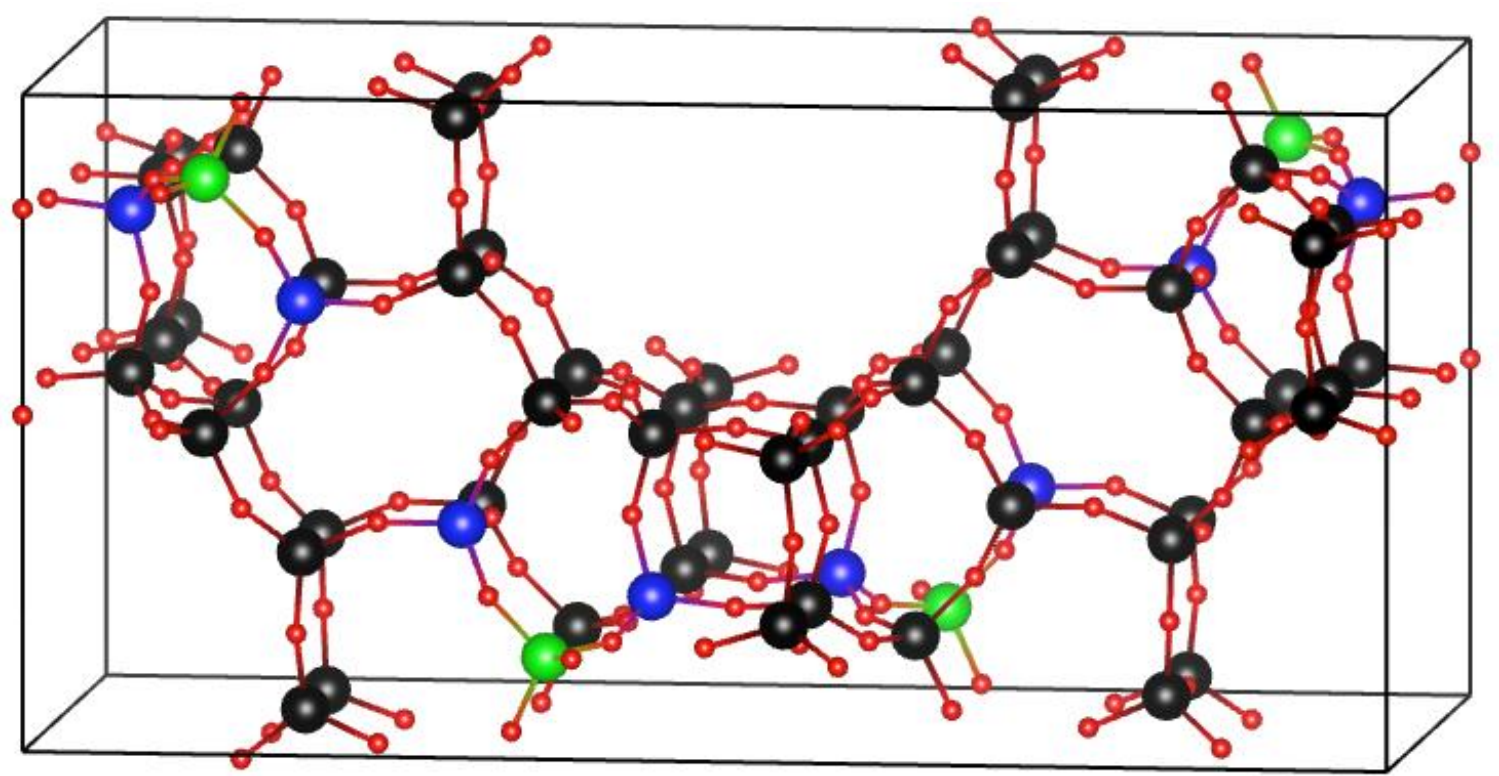


Figure 2

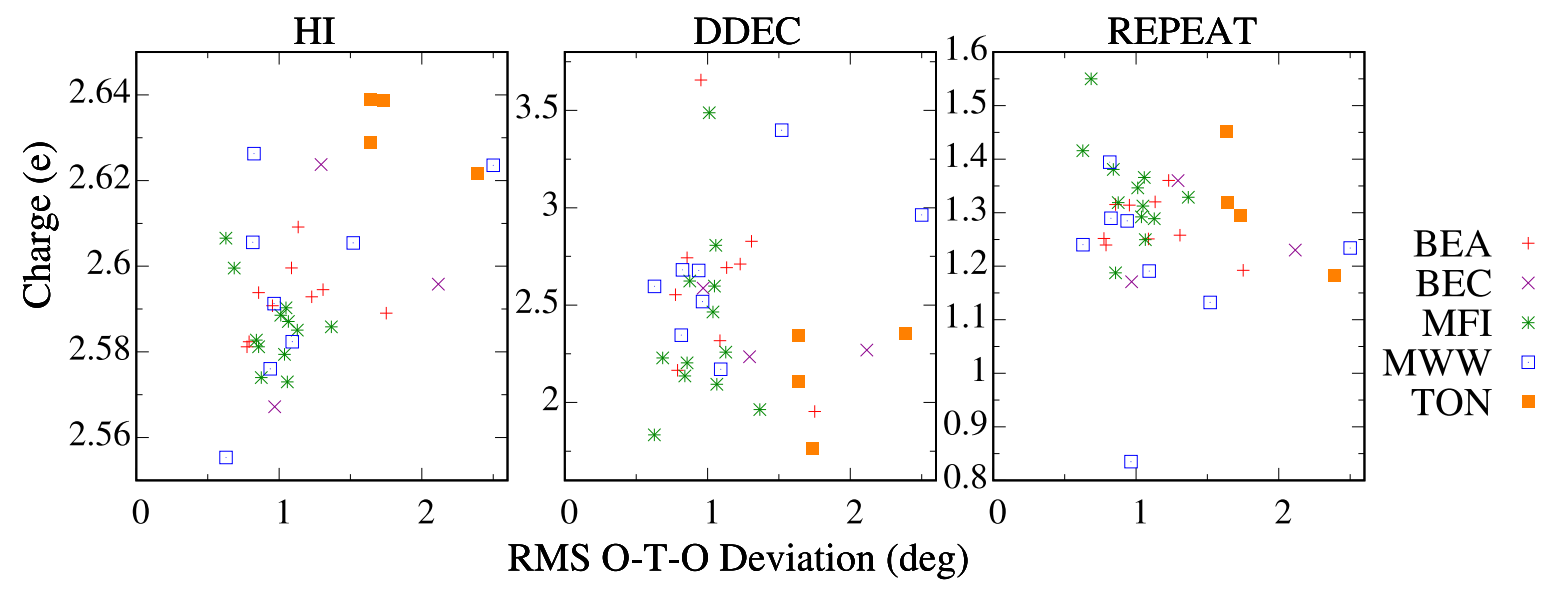


Figure 3

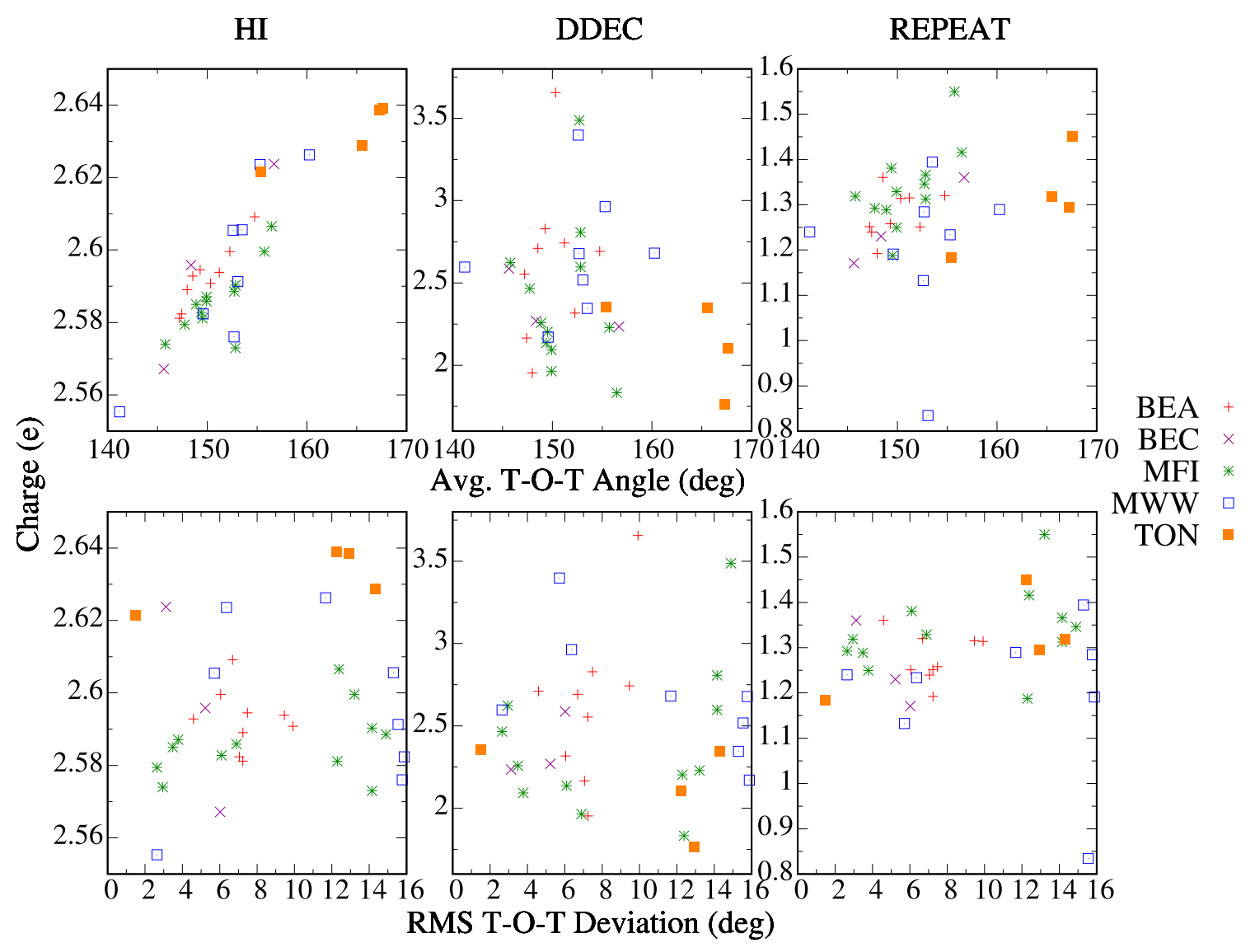


Figure 4

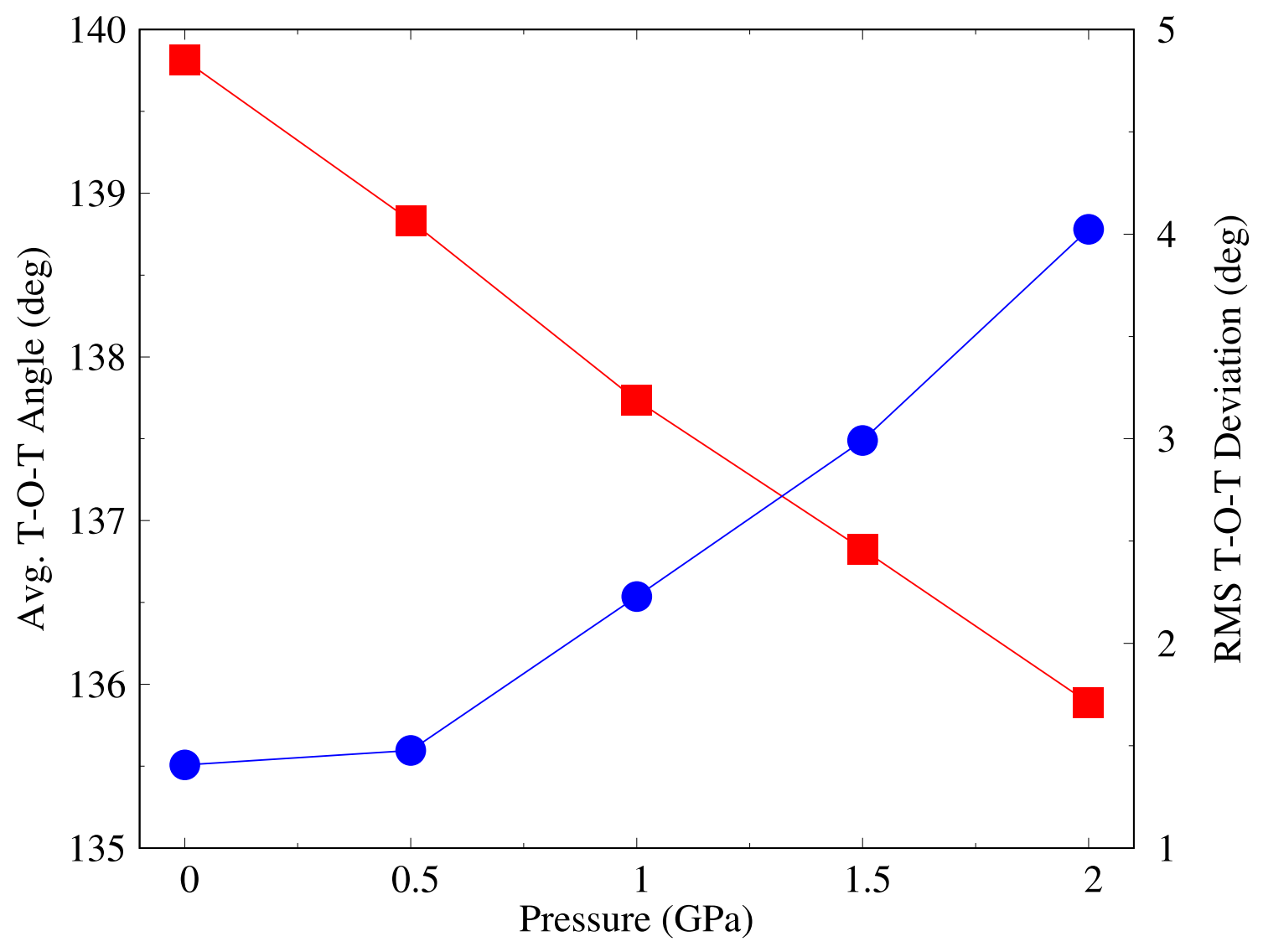


Figure 5

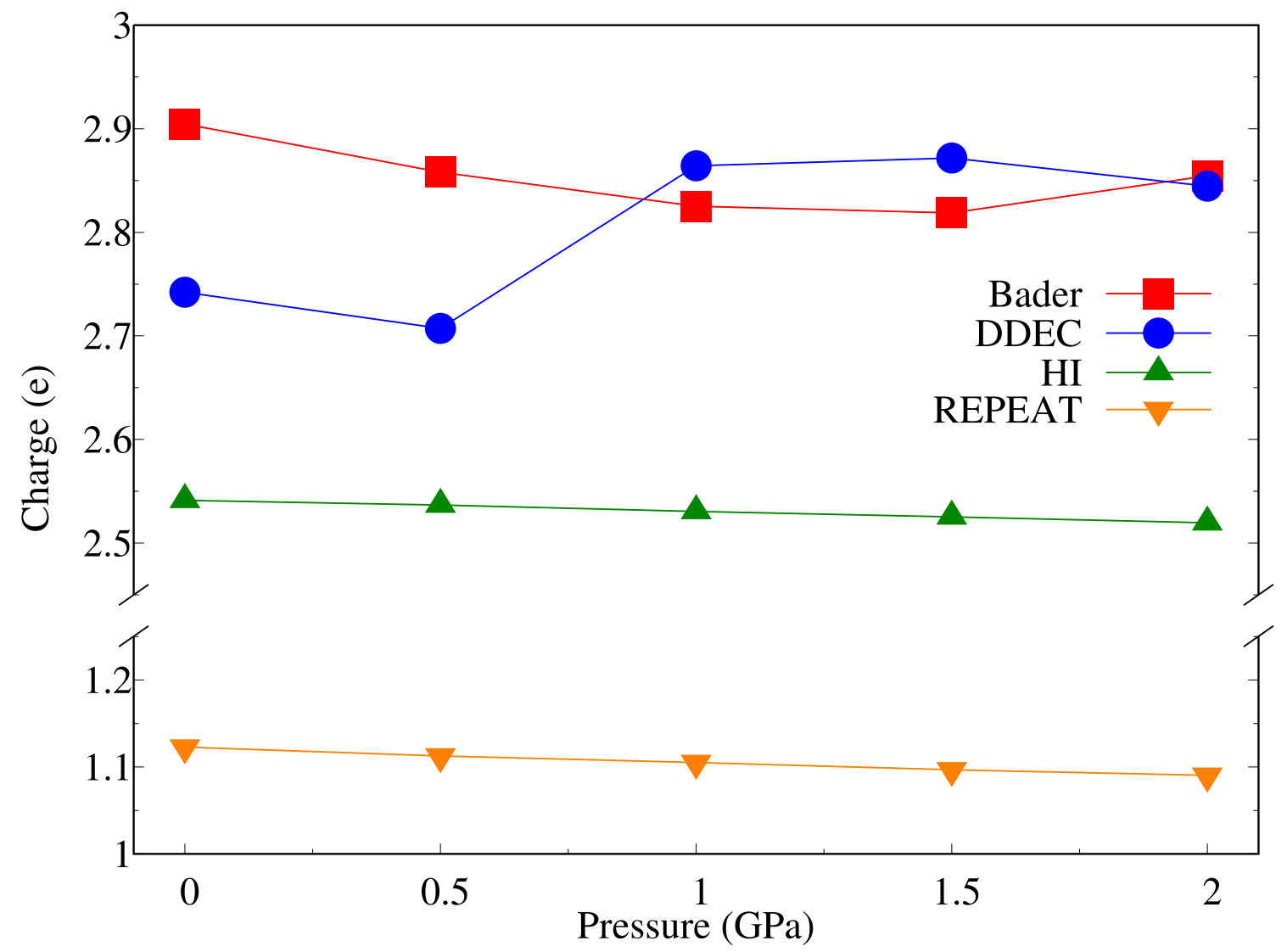


Figure 6
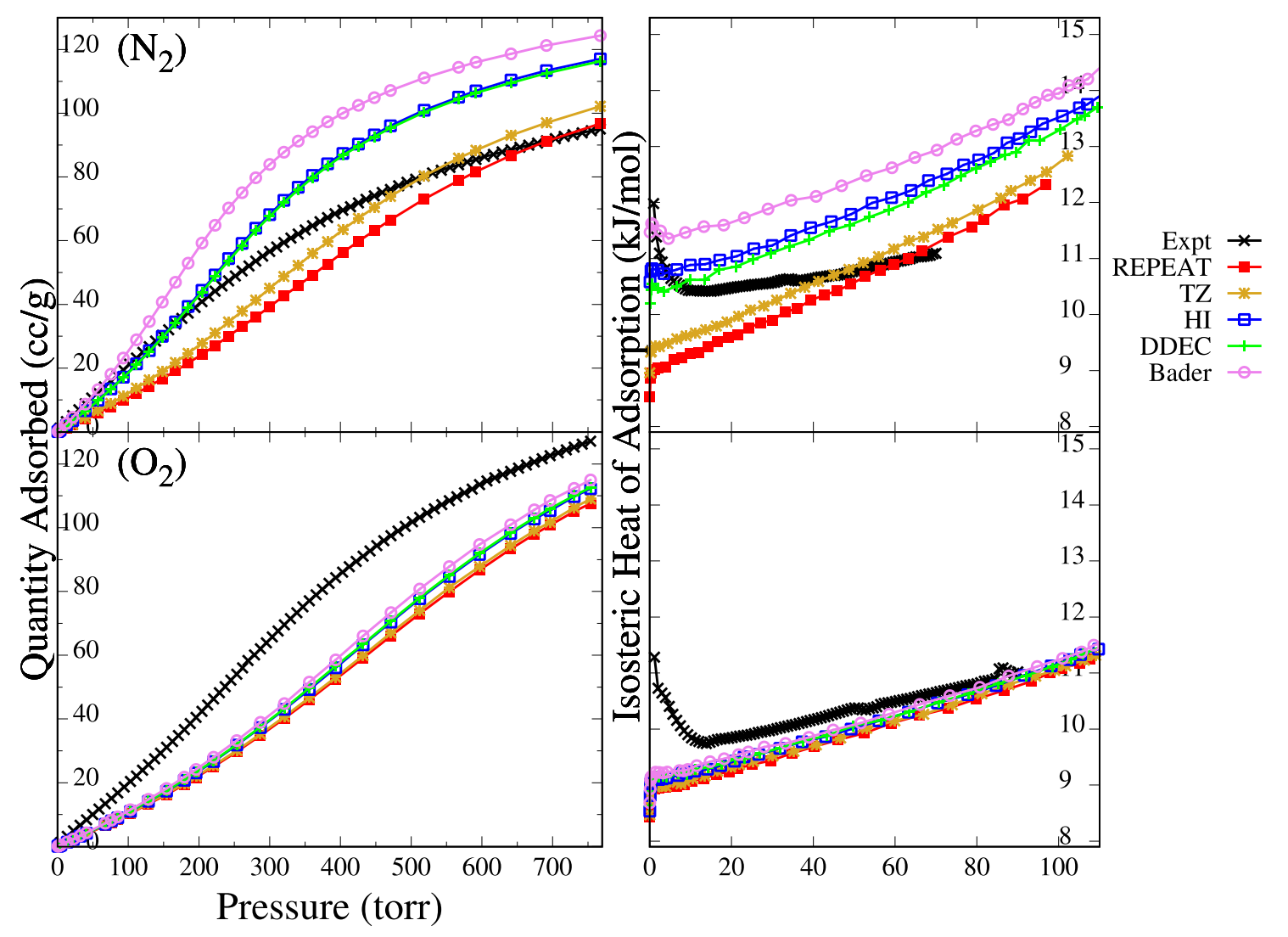
Figure 7
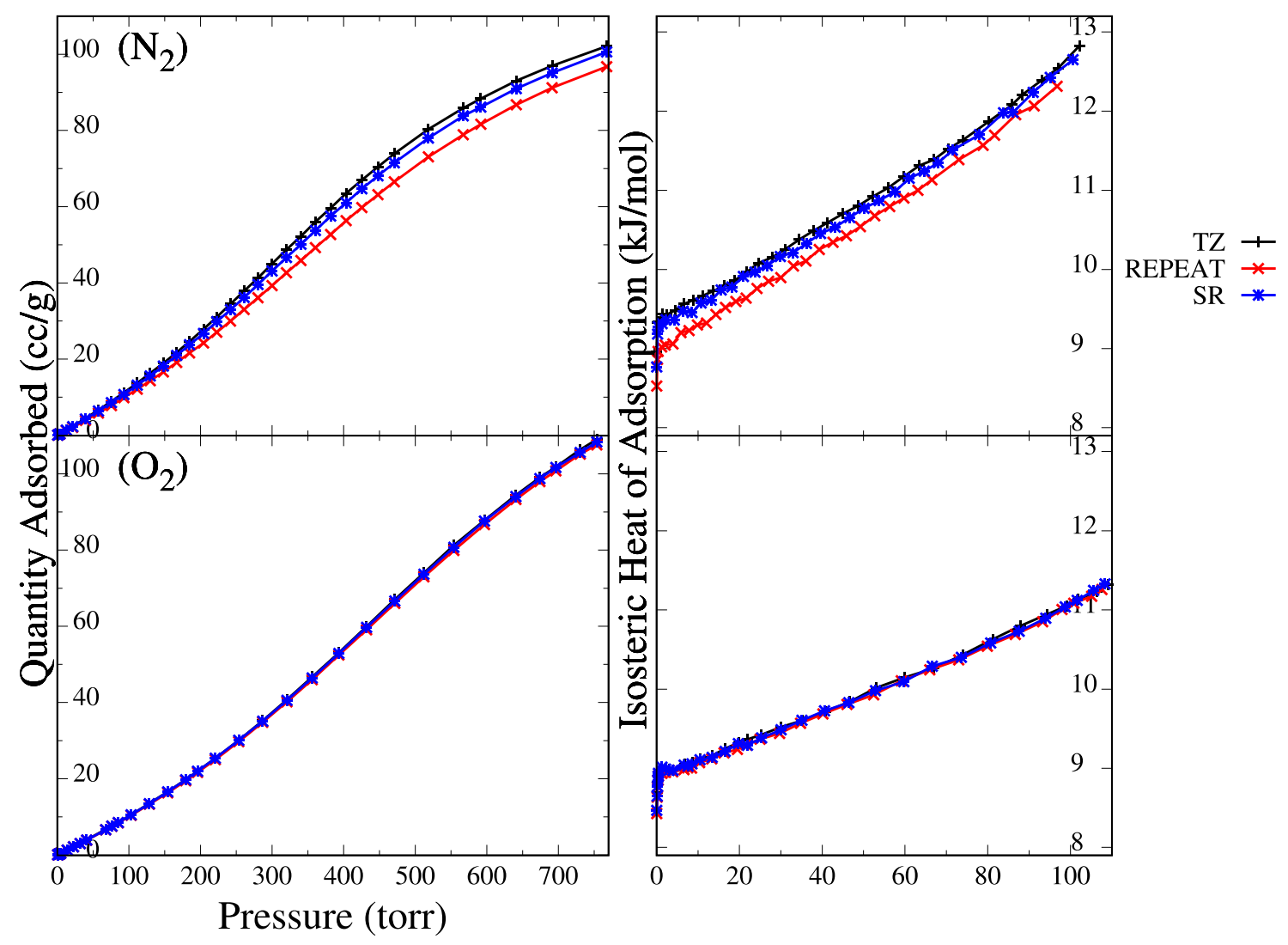